\title{
ON THE APPROXIMATION OF FRONT PROPAGATION PROBLEMS WITH NONLOCAL TERMS
}

\author{
Pierre Cardaliaguet $^{1}$ and Denis Pasquignon ${ }^{2}$
}

\begin{abstract}
We investigate the approximation of the evolution of compact hypersurfaces of $\mathbb{R}^{N}$ depending, not only on terms of curvature of the surface, but also on non local terms such as the measure of the set enclosed by the surface.
\end{abstract}

Mathematics Subject Classification. 65M12, 35K22.

Received: May 5, 2000. Revised: November 8, 2000.

\section{INTRODUCTION}

In this paper, we study a convergence condition of approximation schemes to the evolution of compact, orientated hypersurfaces $\Gamma_{t}=\partial K_{t}$ of $\mathbb{R}^{N}$ moving according to the general law:

$$
V=h\left(x, K_{t}, \nu_{x}, A_{x}\right)
$$

where $V$ is the normal outward velocity of $\Gamma_{t}$ at the point $(t, x), h$ is some evolution law depending on the position $x$, on the outward normal $\nu_{x}$ to $\Gamma_{t}$ at $x \in \Gamma_{t}$, on the curvature ${ }^{a} A$ of $\Gamma_{t}$ at $x$ and on the set $K_{t}$ enclosed by $\Gamma_{t}$.

These motions are generalizations of the famous motion by mean curvature

$$
V=\mathbf{k}_{x}
$$

where $\mathbf{k}_{x}=\operatorname{Trace}\left(A_{x}\right)$ is the mean curvature.

In image processing, the mean curvature motion arises very naturally in order to get a smoothing effect (see [1]). Motions with nonlocal terms also appear: for instance, in [23], the evolution of the front is of nonlocal nature. This motion formalizes a thinning of the initial shape and intends to compute a kind of "skeleton".

Another example is the following evolution law: $h=\operatorname{Trace}\left(A_{x}\right)+\alpha+\beta\left|K_{t}\right|$, studied by Chen, Hilhorst and Logak in [13]:

$$
V=\mathbf{k}_{x}+\alpha+\beta\left|K_{t}\right|
$$

Keywords and phrases. Front propagation, thinning.

1 Université de Bretagne Occidentale, Faculté des Sciences et Techniques, Département de Mathématiques, 6 Avenue Victor Le Gorgeu, BP 80929285 Brest Cedex, France. e-mail: Pierre.Cardaliaguet@univ-brest.fr

2 Centre de recherche CEREMADE, Université Paris IX Dauphine, Place du Maréchal de Lattre de Tassigny, 75775 Paris

Cedex 16, France. e-mail: pasquig@ceremade.dauphine.fr

${ }^{a}$ The curvature is a $(N-1) \times(N-1)$ symmetric matrix, negative if the set is convex. 
where $\left|K_{t}\right|$ is the volume enclosed by $\Gamma_{t}$. This motion is strongly related with the asymptotic behavior of some reaction-diffusion. More generally, the asymptotics of reaction-diffusion systems with non local terms yield front propagation problems of the same nature (see in particular the references of the survey paper [16]).

The main difficulty in studying such evolutions is that they give rise to singularities. In order to define the front after the onset of singularities, several approaches have been proposed. One of the most interesting in a numerical point of view is the level set approach suggested by Osher and Sethian [22]. It amounts to define the solution of the front propagation problem as a level set of the solution of some parabolic p.d.e. Since this p.d.e. is degenerate, it has to be interpreted in the viscosity sence. A rigorous justification of this approach has been done by Evans and Spruck [17], by Chen et al. [12] and Giga et al. [18] who extended it to more general motions of the form

$$
V=h\left(t, x, \nu_{x}, A_{x}\right) .
$$

The key assumption for this approach is that the motion $h$ is parabolic, that is, $h(A) \leq h(B)$ if $A \leq B$. This requirement implies the conservation of the inclusion of the sets enclosed by the hypersurfaces through their evolution: namely

$$
\text { if } K_{0}^{1} \subset K_{0}^{2}, \quad \text { then } K_{t}^{1} \subset K_{t}^{2},
$$

where $K_{t}^{i}$ is the set enclosed by the hypersurface $\Gamma_{t}^{i}$ (see [12]). We call this conservation of the inclusion the "inclusion principle". When $h$ contains a nonlocal terms, the inclusion principle still remains true if $h$ is non decreasing with respect to $K_{t}(c f$. [9]). But if this condition on $h$ is not satisfied, the inclusion principle is not true, therefore the level set approach has to be forsaken. This is the case of the motion in [23] for instance or in (1) with $\beta<0$. Moreover, even if $h$ is non decreasing with respect to the set $K$, the nonlocal parabolic equation given by the level-set approach has a singular kernel, which makes its study difficult with the usual viscosity techniques.

In order to solve this problem, the first author, after adapting the definition of generalized motions given in $[5,6,26]$ to this more general situation, proves in [9]:

1. that there exists a generalized solution when $h$ is non decreasing with respect to $K$ (that is, when the inclusion principle holds);

2. that, even if $h$ enjoys no monotonicity with respect to $K$, but is sufficiently "regular", and if there is a smooth solution to the problem on some interval of time $[0, T]$, then this solution is unique on this interval. Namely, any generalized solution coincides with this solution on the interval.

When $h$ is non decreasing with respect to $K$, the solution is in general not unique. However, uniqueness is generic in some sense (see [9]). There are several results of existence of smooth solutions in short time in the literature (see again [16]). However, the question of existence of generalized solutions is almost completely open when $h$ is not increasing with respect to $K$.

In this paper, we investigate the numerical approximation of the solution of the front propagation problem. Our main goal is to define some consistency conditions for an approximation scheme in order that it converges to the solution. An approximation scheme $\mathbf{T}_{h}$ is a map from the set of compact sets to itself where $h$ is a scale parameter. Given a compact set $K_{0}$, we iterate the operator $\mathbf{T}_{h}$, and we note

$$
K_{h}(n h)=\mathbf{T}_{h}^{n}\left(K_{0}\right) .
$$

Our main results are the following: Given an approximation scheme $\mathbf{T}_{h}$ satisfying some consistency conditions (see Def. 3.1), we have

1. if $h\left(x, K, \nu_{x}, A\right)$ is non decreasing with respect to $K$ and if there is uniqueness of the solution, then $K_{h}(n h)$ converges to the solution at time $t$ when $n h \rightarrow t, n \rightarrow \infty, h \rightarrow 0$,

2. even if $h\left(x, K, \nu_{x}, A\right)$ is not non decreasing with respect to $K$, but if there is a smooth solution to the problem on some interval $[0, T]$, and if $h$ satisfies some regularity conditions, then $K_{h}(n h)$ converges to the solution at time $t$ when $n h \rightarrow t, n \rightarrow \infty, h \rightarrow 0$. 
We complete the paper by giving an explicit approximation scheme for the thinning problem described in [23] and for equation (1).

Our work is inspired by the ideas of Barles and Souganidis [4]. In this work, the authors give conditions guaranteeing the convergence of approximation schemes for fully nonlinear second order equations. Within the framework of viscosity theory, they proved that any monotone, stable and consistent scheme converges to the solution of the problem provided that there exists a comparison principle for this problem.

This result is used to prove the convergence for a wide class of approximations schemes. For instance, this is the method of Barles and Georgelin [3] for proving the convergence of the algorithms developed by Osher and Sethian [22] for approximating the viscosity solution of $u_{t}+F\left(\mathbf{k}_{x}\right) H(D u)=0$ with $F$ a non increasing function. More general schemes are given in Crandall and Lions [15], and their convergence is proved by the same method. To approach the viscosity solutions, we can also use inf-sup schemes, see [11] (for the mean curvature motion), [10,19] (for affine invariant scale space motion), [24] (for general motion depending on the curvature). In image processing, these inf-sup schemes are quite important because they have some invariance properties, see [19]. In particular, all of them are monotone, stable and consistent.

One of the most interesting features of these schemes is the fact that they only require $L^{\infty}$ estimates in order to establish the convergence. The schemes proposed here enjoy a similar property. The price to pay for getting this is that we have to restrict ourselves to a particular class of dynamics. First the map $h(x, K, \nu, A)$ has to be defined whatever $x, K, \nu_{x}$ and $A$. Second either the map $h$ is non decreasing with respect to the set $K$, or satisfies some regularity properties in a neighborhood of smooth sets (for the Hausdorf topology). Let us point out that these requirements rule out many interesting examples of non local evolution equations. In particular, the method developed here cannot be applied to approximate the volume preserving mean curvature motions, the solutions of Hele-Shaw problems or of Mullins-Sekerka models (see [16]). However, several non local equations satisfy our requirements: Typical examples are equation (1) and the thinning equation (17) below.

For adapting Barles-Souganidis method to our problem we are faced with two difficulties. The first one is the non local character of our equations. The second one is the fact that the schemes cannot in general satisfy a comparison principle, because the evolution equations under study usually don't. In order to point out the differences between Barles-Souganidis approach and our, let us now briefly describe the method used in this paper.

Given an approximation scheme $\mathbf{T}_{h}$ and an initial condition $K_{0}$, we construct the approximate solution $K_{h}(n h)$ as described above. As $n h \rightarrow t, h \rightarrow 0$, we can define two limits (in a suitable sense), the lower limit $\mathcal{K}_{*}(t)$ and the upper limit $\mathcal{K}^{*}(t)$. Of course, $\mathcal{K}_{*}(t) \subset \mathcal{K}^{*}(t)$. The problem amounts to prove that $\mathcal{K}_{*}(t)$ and $\mathcal{K}^{*}(t)$ are both equal to the solution, denoted by $\mathcal{K}(t)$ (when it is unique). In Barles-Souganidis approach, it is enough to prove that $\mathcal{K}_{*}$ is a super-solution, while $\mathcal{K}^{*}$ is a sub-solution. Then the comparison principle gives the result.

Here we cannot do so because of the non local character of the equation. Indeed, $\mathcal{K}_{*}$ and $\mathcal{K}^{*}$ do not satisfy the same equation. To overcome this difficulty, we adapt to our geometrical framework the notion of sub/super pair of solutions introduced in [13]: Roughly speaking, we say that $\left(\mathcal{K}_{1}(t), \mathcal{K}_{2}(t)\right)$ is a sub/super pair of solutions, if the time dependent hypersurface $\partial \mathcal{K}_{1}(t)$ is moving according to the law

$$
V^{(1)} \leq \inf _{\mathcal{K}_{1}(t) \subset K \subset \mathcal{K}_{2}(t)} h\left(x, K, \nu_{x}^{(1)}, A_{x}^{(1)}\right)
$$

where $V^{(1)}, \nu_{x}^{(1)}$ and $A_{x}^{(1)}$ are respectively the velocity, the normal and the curvature to $\partial \mathcal{K}_{1}(t)$ at $x$, while the hypersurface $\partial \mathcal{K}_{2}(t)$ is moving according to the law

$$
V^{(2)} \geq \sup _{\mathcal{K}_{1}(t) \subset K \subset \mathcal{K}_{2}(t)} h\left(x, K, \nu_{x}^{(2)}, A_{x}^{(2)}\right)
$$


Our main result (Th. 3.2) is the following: If $\left(\mathcal{K}_{1}(t), \mathcal{K}_{2}(t)\right)$ is a sub/super pair of solutions and if the scheme satisfies a consistency property (see Definition 3.1), then the upper limit $\mathcal{K}^{*}(t)$ and the lower limit $\mathcal{K}_{*}(t)$ are trapped between $\mathcal{K}_{1}(t)$ and $\mathcal{K}_{2}(t)$ :

$$
\mathcal{K}_{1}(t) \subset \mathcal{K}_{*}(t) \subset \mathcal{K}^{*}(t) \subset \mathcal{K}_{2}(t)
$$

These inclusions are derived from a "strengthened inclusion principle".

Therefore, it is interesting to know whether there are such a sub/super pair of solutions which is sufficiently close to the true solution (when it is unique). We prove that this is true in two cases: when there is a smooth solution to the evolution problem, and when the map $h$ is non decreasing with respect to the subset $K$ and there is uniqueness of the solution.

Our paper is divided in two parts: In part one, we first recall several notations, definitions and results of [9]. Then we introduce the new notion of sub/super pairs of solutions of a propagation problem and prove the existence of extremal ones. We also prove that, if there is a smooth solution to the front propagation problem, then the extremal pair of solutions converges to this solution. Finally, we give the "strengthened inclusion principle".

The second part of the paper deals with the convergence of the approximation scheme. After defining the consistency of a scheme, we prove that consistency implies inclusion (2) and, consequently, the convergence of the scheme in some cases. We complete the paper by two examples of explicit approximation schemes.

\section{Sub/SUPER PAir OF SOlutions}

\subsection{Review}

We fix here the notations used throughout the paper. We introduce the notion of sub/super pair of solutions. Although these notions are new in such a context, the main definitions and results come from [9]. So we omit the proofs as far as possible.

For technical reasons, instead of working with hypersurfaces and open sets enclosed by these hypersurfaces, we work with arbitrary bounded subsets of $\mathbb{R}^{N}$, denoted by $\mathcal{K}(t)$, and the desired moving hypersurfaces are the sets $\Gamma_{t}=\partial \mathcal{K}(t)$.

This yields us to work with subsets $\mathcal{K}$ of $\mathbb{R}^{+} \times \mathbb{R}^{N}$. We denote by $(t, x)$ an element of such a set, where $t \in \mathbb{R}^{+}$denotes the time and $x \in \mathbb{R}^{N}$ denotes the space. We set

$$
\mathcal{K}(t)=\mathcal{K} \cap\left(\{t\} \times \mathbb{R}^{N}\right)
$$

and we consider $\mathcal{K}(t)$ as a subset of $\mathbb{R}^{N}$. The closure of the complementary of $\mathcal{K}$ is denoted by $\widehat{\mathcal{K}}$ :

$$
\widehat{\mathcal{K}}=\overline{\left(\mathbb{R}^{+} \times \mathbb{R}^{N}\right) \backslash \mathcal{K}}
$$

and we set

$$
\widehat{\mathcal{K}}(t)=\widehat{\mathcal{K}} \cap\left(\{t\} \times \mathbb{R}^{N}\right) .
$$

As before, $\widehat{\mathcal{K}}(t)$ is considered as a closed subset of $\mathbb{R}^{N}$. 

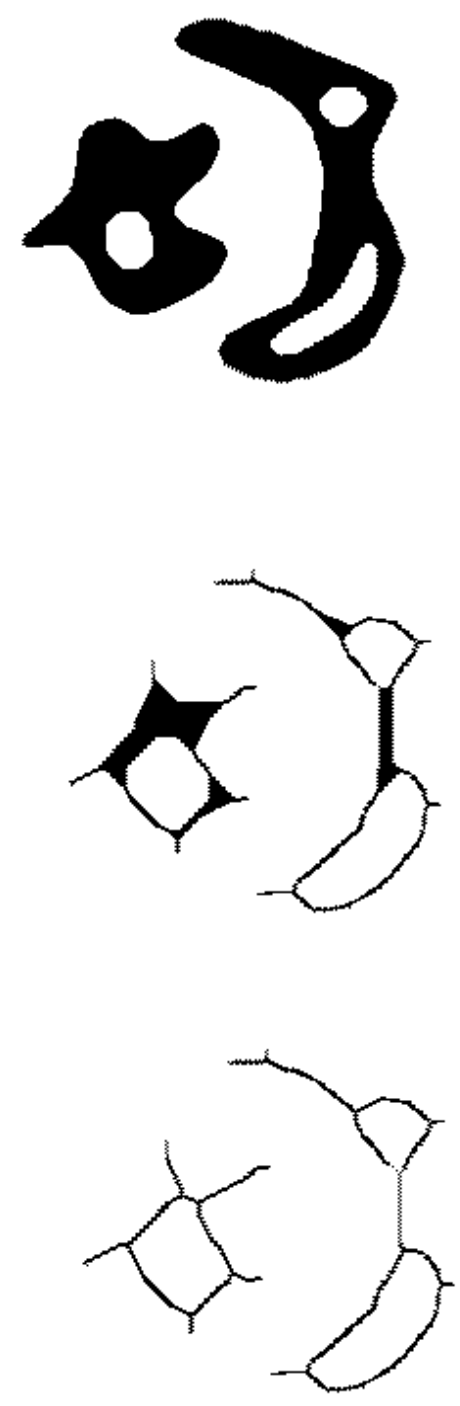

Figure 1. The initial shape is above, we have the evolution at $t=5$ and at $t=10$.

We are seeking the solution of the propagation problem in the set of tubes of $\mathbb{R}^{N}$ :

Definition 2.1 (Tubes of $\mathbb{R}^{N}$ ). A subset $\mathcal{K}$ of $\mathbb{R}^{N+1}$ is a tube if

$$
\forall t>0, \overline{\mathcal{K}(t)} \text { is a compact subset of } \mathbb{R}^{N} \text {. }
$$

The tube is closed if it is closed in $\mathbb{R}^{N+1}$.

We continue with our list of notations: If $E$ is some subset of some finite dimensional space $\mathbb{R}^{p}$ and if $x$ belongs to $\mathbb{R}^{p}$, with denote by $d_{E}(x)$ the distance from $x$ to $E$ :

$$
d_{E}(x)=\inf _{y \in E}\|y-x\|
$$


If $\phi: \mathbb{R}^{+} \times \mathbb{R}^{N} \rightarrow \mathbb{R}$ is a smooth function, we denote by $\phi_{x}(t, x)$ and $\phi_{x x}(t, x)$ the first and second order partial derivatives of $\phi$ with respect to $x$, and by $\phi_{t}(t, x)$ the first order derivative of $\phi$ with respect to $t$. The set of symmetric $N \times N$ matrices is denoted by $\mathcal{S}_{N}$.

Let us set, for any $p \neq 0$ and $X \in \mathcal{S}_{N}$,

$$
H(x, \mathcal{K}(t), p, X)=-\|p\| h\left(x, \mathcal{K}(t), \frac{p}{\|p\|},-\frac{X_{\mid p^{\perp}}}{\|p\|}\right)
$$

where $X_{\mid p^{\perp}}$ is the restriction of $X$ to the subspace $(p)^{\perp}$.

Definition 2.2. Let $\mathcal{B}$ be the set of bounded subsets of $\mathbb{R}^{N}$ and $\mathcal{S}_{N}$ be the set of $N \times N$ symmetric matrices. We say that the map $H: \mathbb{R}^{N} \times \mathcal{B} \times \mathbb{R}_{*}^{N} \times \mathcal{S}_{N} \rightarrow \mathbb{R}$ is geometric if

$$
\forall \lambda \geq 0, H(x, K, \lambda p, \lambda X)=\lambda H(x, K, p, X)
$$

and

$$
H\left(x, K, p,\left(I-\frac{p^{t} p}{\|p\|^{2}}\right) X\left(I-\frac{p^{t} p}{\|p\|^{2}}\right)\right)=H(x, K, p, X)
$$

and $H$ is elliptic if

$$
\forall(X, Y) \in \mathcal{S}_{N}, \quad X \leq Y \Rightarrow H(x, K, p, X) \leq H(x, K, p, Y) .
$$

Let us point out that the map defined by (3) is geometric.

Finally, we set, for $x \in \mathbb{R}^{N}, p \in \mathbb{R}^{N}, X \in \mathcal{S}_{N}$ and $\mathcal{K}$ a tube of $\mathbb{R}^{+} \times \mathbb{R}^{N}$,

$$
\begin{aligned}
H_{*}(x, \mathcal{K}(t), p, X)=\liminf _{\substack{\left(t^{\prime}, x^{\prime}, p^{\prime}, X^{\prime}\right) \\
\\
\epsilon>0, K^{\prime}}} H\left(x^{\prime}, K^{\prime}, p^{\prime}, X^{\prime}\right) \\
\end{aligned}
$$

where this limit is taken over the $\left(t^{\prime}, x^{\prime}, p^{\prime}, X^{\prime}\right) \rightarrow(t, x, p, X), p \neq 0, \epsilon \rightarrow 0^{+}$, and $K^{\prime}$ compact subset of $\mathbb{R}^{N}$ with

$$
\mathcal{K}\left(t^{\prime}\right)-\epsilon B \subset K^{\prime} \subset \mathcal{K}(t)+\epsilon B
$$

where

$$
K+\epsilon B=\left\{x \in \mathbb{R}^{N} \mid d_{K}(x) \leq \epsilon\right\}
$$

and

$$
K-\epsilon B=\left\{x \in K \mid d_{\partial K}(x) \geq \epsilon\right\} .
$$

In the same way, we define

$$
H^{*}(x, \mathcal{K}(t), p, X)=\limsup _{\substack{\left(t^{\prime}, x^{\prime}, p^{\prime}, X^{\prime}\right) \\ \epsilon>0, K^{\prime}}} H\left(x^{\prime}, K^{\prime}, p^{\prime}, X^{\prime}\right)
$$

where this limit is also taken over the $\left(t^{\prime}, x^{\prime}, p^{\prime}, X^{\prime}\right) \rightarrow(t, x, p, X), p \neq 0, \epsilon \rightarrow 0^{+}$, and $K^{\prime}$ compact subset of $\mathbb{R}^{N}$ with

$$
\mathcal{K}\left(t^{\prime}\right)-\epsilon B \subset K^{\prime} \subset \mathcal{K}(t)+\epsilon B .
$$


Note that, if $\mathcal{K}$ is a fixed tube, then $(t, x, p, X) \rightarrow H_{*}(x, \mathcal{K}(t), p, X)$ is l.s.c. while $(t, x, p, X) \rightarrow H^{*}(t, x, \mathcal{K}(t), p, X)$ is u.s.c.

We are now ready to state the definition of a front:

Definition 2.3. Let $K_{0}$ be a compact subset of $\mathbb{R}^{N}$ (the initial condition).

i) A tube $\mathcal{K}$ satisfies the external condition if: $\forall(t, x) \in \partial \mathcal{K}$, with $t>0$, if $\phi \in \mathcal{C}^{2}\left(\mathbb{R}^{+} \times \mathbb{R}^{N}\right)$ has a local maximum on $\overline{\mathcal{K}}$ at $(t, x)$, then

$$
H_{*}\left(x, \mathcal{K}(t), \phi_{x}(t, x), \phi_{x x}(t, x)\right) \leq \phi_{t}(t, x)
$$

and it satisfies the external initial condition for $K_{0}$ if $\mathcal{K}(0)=K_{0}$.

ii) A tube $\mathcal{K}$ satisfies the internal condition if: $\forall(t, x) \in \partial \widehat{\mathcal{K}}, t>0$, if $\phi \in \mathcal{C}^{2}\left(\mathbb{R}^{+} \times \mathbb{R}^{N}\right)$ has a local maximum on $\widehat{\mathcal{K}}$ at $(t, x)$, then

$$
H^{*}\left(x, \mathcal{K}\left(t^{\prime}\right),-\phi_{x}(t, x),-\phi_{x x}(t, x)\right) \geq-\phi_{t}(t, x)
$$

and it satisfies the internal initial condition for $K_{0}$ if $\widehat{\mathcal{K}}(0) \subset \overline{\mathbb{R}^{N} \backslash K_{0}}$.

iii) A tube is a solution to the front propagation problem for $K_{0}$ if it satisfies the internal and the external conditions and the internal and the external initial conditions for $K_{0}$.

Before recalling the main results of [9], let us introduce some assumptions on $H$.

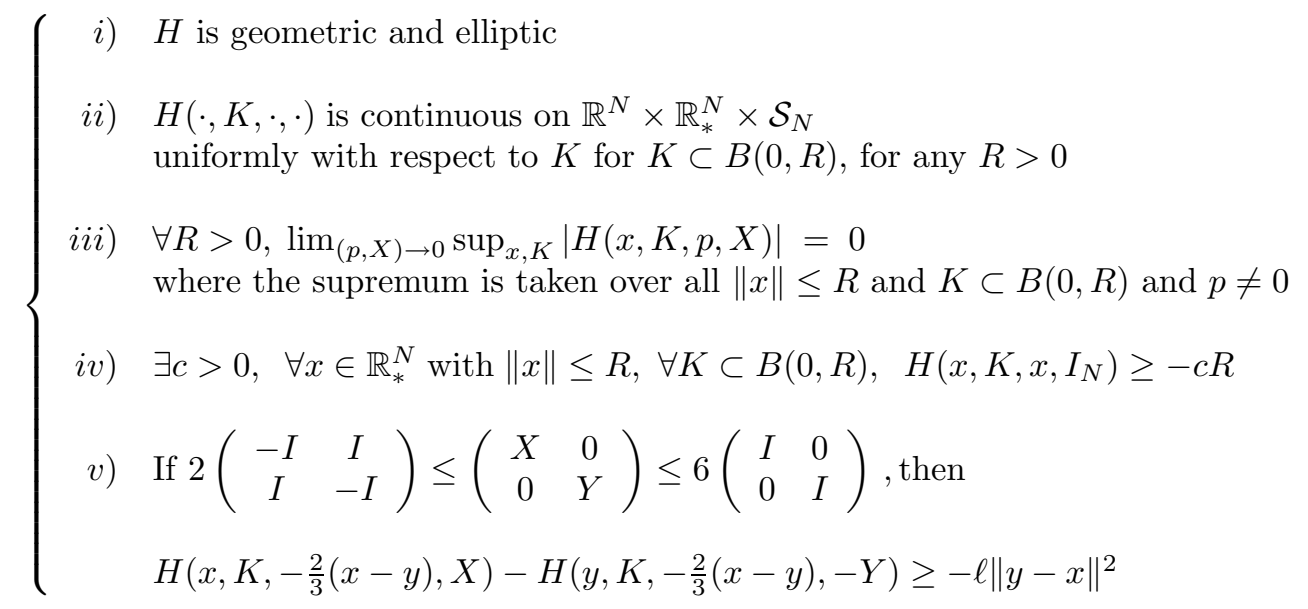

\section{Examples:}

1. If $H$ is of the form

$$
H(x, K, p, X)=\operatorname{Trace}\left(\sigma^{T}\left(X_{\left.\right|_{p}}\right) \sigma\right)+\|p\| \int_{K} \phi\left(x, y, \frac{p}{\|p\|}\right) \mathrm{d} y
$$

where $\sigma=\sigma(x) \in \mathcal{C}^{2}\left(\mathbb{R}^{N}, \mathbb{R}^{(N-1) \times q}\right.$ ) (for some $q$ ), $\phi \in \mathcal{C}_{c}^{1}\left(\mathbb{R}^{3 N}, \mathbb{R}\right.$ ) (not necessarily positive), then $H$ satisfies (6).

2. Example given by (1) satisfies the above conditions when $\beta \leq 0$. The function $H$ defined from $h$ by (3) is the following:

$$
H(K, p, X)=\operatorname{Trace} X_{\left.\right|_{p \perp}}-(\alpha+\beta|K|)\|p\|
$$


In [9], the following results are proved:

\section{Proposition 2.4.}

1. If $H$ satisfies assumptions (6) and is non increasing with respect to $K$, then, for any initial position $K_{0}$, there is a maximal solution to the front propagation problem, i.e., a solution which contains any other solution to the problem, and there is a minimal solution to the front propagation problem, i.e., a solution which is contained in any other solution to the problem. The maximal solution is denoted by $S\left(K_{0}\right)$ while the minimal solution is denoted by $s\left(K_{0}\right)$.

2. if $H$ satisfies (6) and some regularity assumptions (assumptions $\mathbf{H 1}$ and $\mathbf{H 2}$ below), and if there is some classical solution (with a $\mathcal{C}^{3}$ regularity) $\mathcal{K}_{r}$ to the front propagation problem on $[0, T]$ for some $T>0$, then any generalized solution to the front propagation problem starting from $K_{0}$ coincides with $\mathcal{K}_{r}$ on $[0, T]$.

Remark. Assumption (6), part (iv) prevents from applying the Proposition to the motion given by (1) when $\beta$ is positive. Actually in this case, one can show without difficulty that the Proposition still holds true on some interval $[0, T)$ such that either $T=+\infty$ or

$$
\lim _{t \rightarrow T^{-}} \operatorname{diam}(\mathcal{K}(t))=+\infty,
$$

where $\operatorname{diam}(K)$ denotes the diameter of a set $K$.

\subsection{Definition and existence of sub/super pair of solutions}

We now introduce the notion of sub/super pair of solutions. To the best of our knowledge, this notion is new in this context. We borrow it from [13] where it is used in an analytical framework.

As explained in the introduction, a couple $\left(\mathcal{K}_{1}, \mathcal{K}_{2}\right)$ of tubes is a sub/super pair of solutions, if, roughly speaking, the hypersurface $\partial \mathcal{K}_{1}$ is moving according to the law

$$
V^{(1)} \leq \inf _{\mathcal{K}_{1}(t) \subset K \subset \mathcal{K}_{2}(t)} h\left(x, K, \nu_{x}^{(1)}, A_{x}^{(1)}\right)
$$

where $V^{(1)}, \nu_{x}^{(1)}$ and $A_{x}^{(1)}$ are respectively the velocity, the normal and the curvature to $\partial \mathcal{K}_{1}(t)$ at $x$, while the hypersurface $\partial \mathcal{K}_{2}$ is moving according to the law

$$
V^{(2)} \geq \sup _{\mathcal{K}_{1}(t) \subset K \subset \mathcal{K}_{2}(t)} h\left(x, K, \nu_{x}^{(2)}, A_{x}^{(2)}\right),
$$

where similarly $V^{(2)}, \nu_{x}^{(2)}$ and $A_{x}^{(2)}$ are respectively the velocity, the normal and the curvature to $\partial \mathcal{K}_{2}(t)$ at $x$.

We are now going to construct a sub/super pair of solutions which is not necessarily smooth. For doing so, let $H$ be defined by (3) and let us introduce, for $K_{1} \subset K_{2}$, the upper and lower motions $H^{b}$ and $H^{\sharp}$ as

$$
H^{\sharp}\left(x, K_{1}, K_{2}, p, X\right)=\sup _{K_{1} \subset K^{\prime} \subset K_{2}} H\left(x, K^{\prime}, p, X\right)
$$

and

$$
H^{\mathrm{b}}\left(x, K_{1}, K_{2}, p, X\right)=\inf _{K_{1} \subset K^{\prime} \subset K_{2}} H\left(x, K^{\prime}, p, X\right) .
$$

Following the definitions of upper and lower regularization already used for a Hamiltonian $H$, we set, for any $\mathcal{K}_{1}$ and $\mathcal{K}_{2}$ tubes such that $\mathcal{K}_{1} \subset \mathcal{K}_{2}$,

$$
\begin{gathered}
H_{*}^{b}\left(x, \mathcal{K}_{1}(t), \mathcal{K}_{2}(t), p, X\right)=\liminf \underset{\left(t^{\prime}, t^{\prime \prime}, x^{\prime}, p^{\prime}, X^{\prime}\right)}{ } H^{b}\left(x^{\prime}, K_{1}^{\prime}, K_{2}^{\prime}, p^{\prime}, X^{\prime}\right) \\
\epsilon, K_{1}^{\prime}, K_{2}^{\prime}
\end{gathered}
$$


where $\left(t^{\prime}, t^{\prime \prime}, x^{\prime}, p^{\prime}, X^{\prime}\right) \rightarrow(t, t, x, p, X)$ with $p^{\prime} \neq 0$ and $\epsilon \rightarrow 0$ and $K_{1}^{\prime}$ and $K_{2}^{\prime}$ are such that

$$
\mathcal{K}_{1}\left(t^{\prime}\right)-\epsilon B \subset K_{1}^{\prime} \subset \mathcal{K}_{1}(t)+\epsilon B \text { and } \mathcal{K}_{2}\left(t^{\prime \prime}\right)-\epsilon B \subset K_{2}^{\prime} \subset \mathcal{K}_{2}(t)+\epsilon B .
$$

In the same way, we define $\left(H^{b}\right)^{*}$ (with a upper limit instead of a lower limit), and $H_{*}^{\sharp}$ and $\left(H^{\sharp}\right)^{*}$.

Definition 2.5. Let $K_{1}$ and $K_{2}$ be two compact subsets of $\mathbb{R}^{N}$ such that

$$
K_{1} \subset \operatorname{Int}\left(K_{2}\right)
$$

We say that a pair of tubes $\left(\mathcal{K}_{1}, \mathcal{K}_{2}\right)$ is a sub/super pair of solutions with initial conditions $\left(K_{1}, K_{2}\right)$ if

1. $\mathcal{K}_{1} \subset \mathcal{K}_{2}$.

2. If a test function $\phi$ has a maximum on $\mathcal{K}_{1}$ at some point $(t, x) \in \partial \mathcal{K}_{1}$, with $t>0$ then

$$
H_{*}^{\sharp}\left(x, \mathcal{K}_{1}(t), \mathcal{K}_{2}(t), \phi_{x}(t, x), \phi_{x x}(t, x)\right) \leq \phi_{t}(t, x)
$$

(we say that $\mathcal{K}_{1}$ satisfies the external condition for $H^{\sharp}$ ).

3 . If a test function $\phi$ has a maximum on $\widehat{\mathcal{K}_{2}}$ at some point $(t, x) \in \partial \widehat{\mathcal{K}_{2}}$, with $t>0$, then

$$
\left(H^{b}\right)^{*}\left(x, \mathcal{K}_{1}(t), \mathcal{K}_{2}(t),-\phi_{x}(t, x),-\phi_{x x}(t, x)\right) \geq-\phi_{t}(t, x)
$$

(we say that $\mathcal{K}_{2}$ satisfies the internal condition for $H^{\mathrm{b}}$ ).

4. $\mathcal{K}_{1}(0)=K_{1}$ (external initial condition) and $\widehat{\mathcal{K}_{2}}(0) \subset \widehat{K_{2}}$ (internal initial condition).

Lemma 2.6. Let $H$ satisfy assumptions (6). Let $K_{1}$ and $K_{2}$ be compact subsets of $\mathbb{R}^{N}$ with

$$
K_{1} \subset \operatorname{Int}\left(K_{2}\right)
$$

and $\left(\mathcal{K}_{1}, \mathcal{K}_{2}\right)$ be a sub/super pair of solutions with initial conditions $\left(K_{1}, K_{2}\right)$. If, on some interval $[0, T)$ with $T>0$, we have

$$
\forall t \in[0, T), \mathcal{K}_{1}(t) \neq \emptyset
$$

then we have: $\forall t \in[0, T), \mathcal{K}_{2}(t) \neq \emptyset$, and

$$
\forall t \in[0, T), \inf _{x \in \overline{\mathcal{K}}_{1}(t), y \in \widehat{\mathcal{K}}_{2}(t)}\|y-x\| \geq \inf _{x \in K_{1}, y \in \widehat{K}_{2}}\|y-x\| e^{-3 / 2 \ell t}
$$

Proof. The proof is exactly the same than that of Theorem 3.1 of [9], so we omit it.

Corollary 2.7. Assume $H$ satisfies assumption (6). Let $K_{1}$ and $K_{2}$ be compact subsets of $\mathbb{R}^{N}$ with

$$
K_{1} \subset \operatorname{Int}\left(K_{2}\right)
$$

and $\left(\mathcal{K}_{1,1}, \mathcal{K}_{1,2}\right)$ and $\left(\mathcal{K}_{2,1}, \mathcal{K}_{2,2}\right)$ be two sub/super pairs of solutions with initial conditions $\left(K_{1}, K_{2}\right)$. Then, if there is some $T>0$ such that

$$
\forall t \in[0, T), \quad \mathcal{K}_{1,1}(t) \neq \emptyset
$$

then we have

$$
\forall t \geq 0, \inf _{x \in \overline{\mathcal{K}}_{1,1}(t), y \in \widehat{\mathcal{K}_{2,2}}(t)}\|y-x\| \geq \inf _{x \in K_{1}, y \in \widehat{K_{2}}}\|y-x\| \mathrm{e}^{-3 / 2 \ell t}
$$


In particular,

$$
\forall t \in[0, T), \mathcal{K}_{1,1}(t) \subset \mathcal{K}_{2,2}(t)
$$

Proof. Let us set

$$
d(t)=\inf _{x \in \overline{\mathcal{K}}_{1,1}(t), y \in \widehat{\mathcal{K}_{2,2}}(t)}\|y-x\|
$$

We fix $\epsilon>0$ and we define by $T^{*}$ the largest time such that

$$
\forall t \in\left[0, T^{*}\right), \quad d(t) \geq(1-\epsilon) d(0) \mathrm{e}^{-3 / 2 \ell t} .
$$

Our aim is to prove that $T^{*}=T$. Note that $T^{*}$ is positive because $\mathcal{K}_{1,1}$ satisfies the external initial condition while $\mathcal{K}_{2,2}$ satisfies the internal initial condition. We assume that, contrary to our claim, $T^{*}<T$. Let us set

$$
\mathcal{K}_{1}=\mathcal{K}_{1,1} \cup \mathcal{K}_{2,1} \text { and } \mathcal{K}_{2}=\mathcal{K}_{1,2} \cap \mathcal{K}_{2,2}
$$

It is easy to check that $\left(\mathcal{K}_{1}, \mathcal{K}_{2}\right)$ is a sub/super pair of solutions with initial conditions $\left(K_{1}, K_{2}\right)$ on the interval $\left[0, T^{*}\right)$. Therefore, from Lemma 2.6 , we have

$$
\forall t \in\left[0, T^{*}\right), \inf _{x \in \mathcal{K}_{1}(t), y \in \widehat{\mathcal{K}}_{2}(t)}\|y-x\| \geq \inf _{x \in K_{1}, y \in \widehat{K_{2}}}\|y-x\| \mathrm{e}^{-3 / 2 \ell t}
$$

Note also that $d(t)$ is left-continuous (see step (3) of the proof of Th. 3.1 of [9]). Thus we have

$$
d\left(T^{*}\right) \geq d(0) \mathrm{e}^{-3 / 2 \ell T^{*}}>(1-\epsilon) d(0) \mathrm{e}^{-3 / 2 \ell T^{*}}
$$

because $d(0)>0$. From the lower semi-continuity of $d$, there is some $\tau>0$ with

$$
\forall t \in\left[0, T^{*}+\tau\right), d(t) \geq(1-\epsilon) d(0) \mathrm{e}^{-3 / 2 \ell t}
$$

This is in contradiction with the definition of $T^{*}$. So, letting $\epsilon \rightarrow 0^{+}$, we have obtained

$$
\forall t \in[0, T), \quad d(t) \geq d(0) \mathrm{e}^{-3 / 2 \ell t}
$$

Theorem 2.8. Assume that the motion $H$ satisfies assumptions (6). Let $K_{1}$ and $K_{2}$ be compact subsets of $\mathbb{R}^{N}$ with

$$
K_{1} \subset \operatorname{Int}\left(K_{2}\right)
$$

Then there is an extremal sub/super pair $\left(\mathcal{K}_{1}^{\mathrm{e}}, \mathcal{K}_{2}^{\mathrm{e}}\right)$ of solutions for $\left(K_{1}, K_{2}\right)$, i.e. a pair that satisfies the following properties:

1. $\left(\mathcal{K}_{1}^{\mathrm{e}}, \mathcal{K}_{2}^{\mathrm{e}}\right)$ is sub/super pair of solutions with initial condition $\left(K_{1}, K_{2}\right)$.

2. If $\left(\mathcal{K}_{1}, \mathcal{K}_{2}\right)$ is another sub/super pair of solutions with initial condition $\left(K_{1}, K_{2}\right)$, then

$$
\mathcal{K}_{1} \subset \mathcal{K}_{1}^{\mathrm{e}} \text { and } \mathcal{K}_{2}^{\mathrm{e}} \subset \mathcal{K}_{2}
$$


Proof.

1. Let $\mathcal{E}$ be the set of sub/super pair of solutions $\left(\mathcal{A}_{1}, \mathcal{A}_{2}\right)$ such that $\mathcal{A}_{1}$ satisfies the external initial condition for some set $\mathcal{A}_{1}(0) \subset K_{1}$ and such that $\mathcal{A}_{2}$ satisfies the internal initial condition for $K_{2}$. Let us set

$$
\mathcal{K}_{1}=\overline{\bigcup_{\left(\mathcal{A}_{1}, \mathcal{A}_{2}\right) \in \mathcal{E}} \mathcal{A}_{1}}
$$

and

$$
\mathcal{K}_{2}=\bigcap_{\left(\mathcal{A}_{1}, \mathcal{A}_{2}\right) \in \mathcal{E}} \mathcal{A}_{2}
$$

If we prove that $\left(\mathcal{K}_{1}, \mathcal{K}_{2}\right)$ is a sub/super pair of solutions, then this pair is clearly extremal. Note that $\mathcal{K}_{1}$ and $\mathcal{K}_{2}$ are tubes from Proposition 3.3 of [9]. Let us now prove that $\left(\mathcal{K}_{1}, \mathcal{K}_{2}\right)$ is a sub/super pair of solutions.

2. We first prove that $\mathcal{E}$ is not empty. Let us set $\mathcal{A}_{1}=\{0\} \times K_{1}$ and

$$
H_{0}(x, K, p, A)=\inf _{K^{\prime} \subset K} H\left(x, K^{\prime}, p, A\right) .
$$

Then $H_{0}$ satisfies assumptions (6) and is non increasing with respect to $K$. Therefore, from Theorem 3.5 of [9], there is some solution $\mathcal{A}_{2}$ to the front propagation problem with initial condition $K_{2}$. With these definitions, the pair $\left(\mathcal{A}_{1}, \mathcal{A}_{2}\right)$ clearly belongs to $\mathcal{E}$.

3. Inclusion

$$
\mathcal{K}_{1} \subset \mathcal{K}_{2}
$$

holds true thanks to Corollary 2.7.

4. We now prove that $\left(\mathcal{K}_{1}, \mathcal{K}_{2}\right)$ is a sub/super pair of solutions. Let $(t, x) \in \partial \mathcal{K}_{1}$, with $t>0$, and $\phi$ be such that $(t, x)$ is a local maximum of $\phi$ on $\mathcal{K}_{1}$. Without loss of generality, we can assume that $(t, x)$ is a strict local maximum (otherwise, we prove the result for $\phi(s, y)+\epsilon\|(s, y)-(t, x)\|^{2}$, which has a strict local maximum on $\mathcal{K}_{1}$ at $(t, x)$, and we let $\left.\epsilon \rightarrow 0^{+}\right)$.

From the very definition of $\mathcal{K}_{1}$, there are $\left(\mathcal{A}_{1, n}, \mathcal{A}_{2, n}\right) \in \mathcal{E}$ such that

$$
\lim _{n \rightarrow+\infty} d_{\mathcal{A}_{1, n}}(t, x)=0
$$

Then, since $(t, x)$ is a strict local maximum of $\phi$, there are $\left(t_{n}, x_{n}\right)$ local maxima of $\phi$ on $\mathcal{A}_{1, n}$ which converge to $(t, x)$, and we have

$$
H_{*}^{\sharp}\left(x_{n}, \mathcal{A}_{1, n}\left(t_{n}\right), \mathcal{A}_{2, n}\left(t_{n}\right), \phi_{x}\left(t_{n}, x_{n}\right), \phi_{x x}\left(t_{n}, x_{n}\right)\right) \leq \phi_{t}\left(t_{n}, x_{n}\right)
$$

because the $\left(\mathcal{A}_{1, n}, \mathcal{A}_{2, n}\right)$ is a sub/super pair of solutions. Since $\mathcal{A}_{1, n} \subset \mathcal{K}_{1}$ and $\mathcal{K}_{2} \subset \mathcal{A}_{2, n}$, we have

$$
H_{*}^{\sharp}\left(x_{n}, \mathcal{K}_{1}\left(t_{n}\right), \mathcal{K}_{2}\left(t_{n}\right), \phi_{x}\left(t_{n}, x_{n}\right), \phi_{x x}\left(t_{n}, x_{n}\right)\right) \leq H_{*}^{\sharp}\left(x_{n}, \mathcal{A}_{1, n}\left(t_{n}\right), \mathcal{A}_{2, n}\left(t_{n}\right), \phi_{x}\left(t_{n}, x_{n}\right), \phi_{x x}\left(t_{n}, x_{n}\right)\right) .
$$

From the lower semi-continuity of the map

$$
(t, x, p, X) \rightarrow H_{*}^{\sharp}\left(x, \mathcal{K}_{1}(t), \mathcal{K}_{2}(t), p, X\right),
$$

we get:

$$
H_{*}^{\sharp}\left(x, \mathcal{K}_{1}(t), \mathcal{K}_{2}(t), \phi_{x}(t, x), \phi_{x x}(t, x)\right) \leq \phi_{t}(t, x)
$$

So $\mathcal{K}_{1}$ satisfies the external condition for $H^{\sharp}$. 
5. We can prove in the same way that, if a test function $\phi$ has a maximum on $\widehat{\mathcal{K}_{2}}$ at some point $(t, x) \in \partial \widehat{\mathcal{K}_{2}}$, then

$$
\left(H^{b}\right)^{*}\left(x, \mathcal{K}_{1}(t), \mathcal{K}_{2}(t),-\phi_{x}(t, x),-\phi_{x x}(t, x)\right) \geq-\phi_{t}(t, x) .
$$

So the pair $\left(\mathcal{K}_{1}, \mathcal{K}_{2}\right)$ satisfies the external and internal conditions required in the definition.

6. It remains to prove that $\mathcal{K}_{1}$ satisfies the external initial condition for $K_{1}$ while $\mathcal{K}_{2}$ satisfies the internal condition for $K_{2}$.

We first prove that $\mathcal{K}_{1}(0)=K_{1}$. From step 2, the inclusion $K_{1} \subset \mathcal{K}_{1}(0)$ holds true. Let us prove the converse inclusion. Let $x_{0} \notin K_{1}$. From Lemma 3.2 of [9], there is $\epsilon>0$ such that, for any tube $\mathcal{A}$ satisfying the external condition for $H$ and the external initial condition for $K_{1}$, we have

$$
d_{\mathcal{A}}\left(0, x_{0}\right) \geq \epsilon
$$

Since $\left(\mathcal{A}_{1}, \mathcal{A}_{2}\right)$ belongs to $\mathcal{E}, \mathcal{A}_{1}$ satisfies the external condition for $H$ and the external initial condition for $K_{1}$. Thus

$$
d_{\mathcal{A}_{1}}\left(0, x_{0}\right) \geq \epsilon .
$$

so that $x_{0} \notin \mathcal{A}_{1}(0)$. Therefore, $\mathcal{A}_{1}$ satisfies the external initial condition for $K_{2}$.

In the same way, using Lemma 3.4 of [9], we can prove that $\mathcal{K}_{2}$ satisfies the internal initial condition for $K_{2}$, i.e., $\widehat{\mathcal{K}_{2}}(0) \subset \overline{\mathbb{R}^{N} \backslash K_{2}}$.

\subsection{Approximation of a motion by a pair of sub/super solutions}

In this section, we investigate the behavior of extremal pair of solutions with initial conditions $\left(K_{0}-\epsilon B, K_{0}+\right.$ $\epsilon B)$ when $\epsilon \rightarrow 0^{+}$. Let us recall that the sets $K_{0}-\epsilon B$ and $K_{0}+\epsilon B$ are defined by (5) and (4).

We are able to prove that these extremal pair of solutions approximate the solution in two cases: First when $H$ is non increasing with respect to $K$ and the solution is unique. Second when there is a smooth solution to the problem.

Let us first assume that $H$ is non increasing with respect to $K$. According to Proposition 2.4, there exist a maximal and a minimal solution to the motion problem : any solution is contained in the maximal solution and contains the minimal one. We recall that one denotes by $S\left(K_{0}\right)$ the maximal solution and by $s\left(K_{0}\right)$ the minimal one with initial condition $K_{0}$.

Proposition 2.9. Assume that $H$ satisfies assumption (6) and is non increasing with respect to $K$. Then, for any $\epsilon>0,\left(S\left(K_{0}-\epsilon B\right), S\left(K_{0}+\epsilon B\right)\right)$ is a pair of sub/super solutions.

Moreover,

$$
\bigcup_{\epsilon>0} S\left(K_{0}-\epsilon B\right)=s\left(K_{0}\right) \text { and } \bigcap_{\epsilon>0} S\left(K_{0}+\epsilon B\right)=S\left(K_{0}\right) .
$$

Therefore, if there is uniqueness of the solution, i.e., if $\overline{s\left(K_{0}\right)}=S\left(K_{0}\right)$; then the extremal pair of sub/super solutions converges to the solution. Let us recall that uniqueness is generic in a suitable sense (see [9]).

Proof. If $H$ is non increasing with respect to $K$, then

$$
H^{\sharp}\left(x, K_{1}, K_{2}, p, A\right)=H\left(x, K_{1}, p, A\right) \text { and } H^{b}\left(x, K_{1}, K_{2}, p, A\right)=H\left(x, K_{2}, p, A\right) .
$$

Thus $\left(S\left(K_{0}-\epsilon B\right), S\left(K_{0}+\epsilon B\right)\right)$ is a pair of sub/super solutions.

From [9], we know that $\bigcap_{\epsilon>0} S\left(K_{0}+\epsilon B\right)=S\left(K_{0}\right)$ because the set-valued map $K_{0} \rightarrow S\left(K_{0}\right)$ is upper semi-continuous and $S\left(K_{0}\right) \subset S\left(K_{0}+\epsilon B\right)$. 
The other equality follows from the very construction of $s\left(K_{0}\right)$ (see Th. 3.9 of [9]).

We now assume that there is a smooth solution to the front propagation problem for some smooth initial set $K_{0}$ on an interval $[0, T]$. Then Proposition 2.4 states that, under some assumptions $(\mathbf{H 1})$ and $(\mathbf{H 2})$ that we recall now, this solution is unique.

(H1) We first assume that the non local term in $H$ is Lipschitz continuous in a neighborhood of smooth compact sets:

If $K$ is a compact set with $\mathcal{C}^{2}$ boundary and if $M>0$, there are $k(K, M)$ and $\alpha(K, M)>0$ such that for any compact set $K^{\prime}$ with

$$
\mathcal{H}\left(K, K^{\prime}\right)+\mathcal{H}\left(\widehat{K}, \widehat{K^{\prime}}\right) \leq \alpha(K, M),
$$

(where $\mathcal{H}$ denotes the Hausdorf distance) we have:

$$
\left|H(x, K, p, X)-H\left(x, K^{\prime}, p, X\right)\right| \leq k(K, M)\left[\mathcal{H}\left(K, K^{\prime}\right)+\mathcal{H}\left(\widehat{K}, \widehat{K}^{\prime}\right)\right]
$$

for any $(x, p, X)$ such that

$$
\|x\| \leq M,\|p\|=1,\|X\| \leq M .
$$

Moreover, the constants $k(K, M)$ and $\alpha(K, M)$ depend continuously on $K$ for the $\mathcal{C}^{2}$ norm.

(H2) We also assume a Lipschitz dependence of $H$ with respect to $x$ and $X$ : For any $M>0$, there some Lipschitz constant $\ell(M)$ such that

$$
\left|H(x, K, p, X)-H\left(x^{\prime}, K, p, X^{\prime}\right)\right| \leq \ell(M)\left[\left\|x-x^{\prime}\right\|+\left\|X-X^{\prime}\right\|\right]
$$

for any $\left(x, x^{\prime}, K, p, X, X^{\prime}\right)$ such that

$$
\left\|\left(x, x^{\prime}\right)\right\| \leq M,\|p\|=1,\left\|X, X^{\prime}\right\| \leq M, \sup _{y \in K}\|y\| \leq M .
$$

Examples. If $H$ is as in (7), then $H$ satisfies not only (6), but also (H1) and (H2). It is also the case for the motion defined by (1), where $H$ is given by (8).

The following Theorem states that, if there is a smooth solution to the front propagation problem, then the extremal sub/super pair of solutions for the initial condition $\left(K_{0}-\epsilon B, K_{0}+\epsilon B\right)$ converges to this solution on $[0, T]$ as $\epsilon \rightarrow 0^{+}$.

Theorem 2.10. Assume that $H$ satisfies assumptions (6), (H1) and (H2). Let $K_{0}$ be a compact subset of $\mathbb{R}^{N}$ with $\mathcal{C}^{3}$ boundary and $\mathcal{K}_{r}$ be a classical solution (with a $\mathcal{C}^{3}$ regularity) to the front propagation starting from $K_{0}$ on $[0, T]$ for some $T>0$.

Let $\left(\mathcal{K}_{1}^{\epsilon}, \mathcal{K}_{2}^{\epsilon}\right)$ be an extremal pair of solutions with initial positions $\left(K_{0}-\epsilon B, K_{0}+\epsilon B\right)$. Then $\mathcal{K}_{1}^{\epsilon}$ and $\mathcal{K}_{2}^{\epsilon}$ converge to $\mathcal{K}_{r}$ on $[0, T]$. Namely, there is a constant $k$ such that, for any $t \in[0, T]$,

$$
\mathcal{K}_{1}^{\epsilon}(t) \subset \mathcal{K}_{r}(t) \subset \mathcal{K}_{1}^{\epsilon}(t)+\epsilon \mathrm{e}^{k t} B
$$

and

$$
\mathcal{K}_{r}(t) \subset \mathcal{K}_{2}^{\epsilon}(t) \subset \mathcal{K}_{r}(t)+\epsilon \mathrm{e}^{k t} B
$$

Proof. The proof is an adaptation of the proof of Theorem 4.8 of [9]. 


\subsection{The strengthened inclusion principle}

We have just proved that, under suitable condition, the extremal sub/super pair of solutions $\left(\mathcal{K}_{1}^{\epsilon}, \mathcal{K}_{2}^{\epsilon}\right)$ starting from $\left(K_{0}-\epsilon B, K_{0}+\epsilon B\right)$ provides a good approximation of the solution to the front propagation problem starting from $K_{0}$. Next we prove that, if $\mathcal{K}^{*}$ and $\mathcal{K}_{*}$ are the upper limit and the lower limit of the approximate motion $K_{h}(n h)$ for some consistent approximation scheme $\mathbf{T}_{h}$, then $\mathcal{K}^{*}$ and $\mathcal{K}_{*}$ are trapped between $\mathcal{K}_{1}^{\epsilon}$ and $\mathcal{K}_{2}^{\epsilon}$ :

$$
\mathcal{K}_{1}^{\epsilon}(t) \subset \mathcal{K}_{*}(t) \subset \mathcal{K}^{*}(t) \subset \mathcal{K}_{2}^{\epsilon}(t)
$$

Letting $\epsilon \rightarrow 0$ gives then the desired convergence result.

For getting inclusions (11), we proceed in two steps: 1) we prove that the limits $\mathcal{K}^{*}$ and $\mathcal{K}_{*}$ satisfy some internal and external conditions and 2) that these conditions imply the desired inclusions. The second step of this program is precisely the aim of Theorem 2.11 below. Step 1 derives from the consistency of the scheme, and we postpone its proof until the next section.

The internal and external conditions derived by step 1 are the following (we are working on some interval of time $[0, T]$ with $T>0)$ :

(C1) $\forall t \in[0, T), \mathcal{K}_{*}(t) \subset \mathcal{K}^{*}(t)$.

(C2) $\mathcal{K}_{*}$ satisfies the internal condition for $H^{\sharp}$ on $[0, T]$ i.e. for any $t \in(0, T)$, if a test function $\phi$ has a maximum on $\widehat{\mathcal{K}_{*}}$ at some point $(t, x) \in \partial \widehat{\mathcal{K}_{*}}$, then

$$
\left(H^{\sharp}\right)^{*}\left(x, \mathcal{K}_{*}(t), \mathcal{K}^{*}(t),-\phi_{x}(t, x),-\phi_{x x}(t, x)\right) \geq-\phi_{t}(t, x) .
$$

(C3) $\mathcal{K}^{*}$ satisfies the external condition for $H^{b}$ on $[0, T)$ i.e. for any $t \in(0, T)$, if a test function $\phi$ has a maximum on $\mathcal{K}^{*}$ at some point $(t, x) \in \partial \mathcal{K}^{*}$, then

$$
H_{*}^{b}\left(x, \mathcal{K}_{*}(t), \mathcal{K}^{*}(t), \phi_{x}(t, x), \phi_{x x}(t, x)\right) \leq \phi_{t}(t, x) .
$$

Under these conditions, we can derive the following

Theorem 2.11. Assume that the motion $H$ satisfies assumptions (6), that the tubes $\mathcal{K}^{*}$ and $\mathcal{K}_{*}$ satisfy the above conditions (C1), (C2) and (C3). Let $\left(\mathcal{K}_{1}, \mathcal{K}_{2}\right)$ be a sub/super pair of solutions, such that $\mathcal{K}_{1}(t)$ and $\mathcal{K}_{2}(t)$ are non empty and bounded for $t \in[0, T]$, for some $T>0$.

If

$$
\overline{\mathcal{K}_{1}}(0) \subset \operatorname{Int}\left(\mathcal{K}_{*}(0)\right) \text { and } \mathcal{K}^{*}(0) \subset \operatorname{Int}\left(\mathcal{K}_{2}(0)\right)
$$

then we have

$$
\forall t \in[0, T), \mathcal{K}_{1}(t) \subset \operatorname{Int}\left(\mathcal{K}_{*}(t)\right) \text { and } \mathcal{K}^{*}(t) \subset \operatorname{Int}\left(\mathcal{K}_{2}(t)\right) .
$$

More precisely, if we set

$$
d_{1}(t)=\inf _{y_{1} \in \mathcal{K}_{1}(t), y_{2} \in \widehat{\mathcal{K}}_{*}(t)}\left\|y_{1}-y_{2}\right\|
$$

and

$$
d_{2}(t)=\inf _{y_{1} \in \mathcal{K}^{*}(t), y_{2} \in \widehat{\mathcal{K}_{2}}(t)}\left\|y_{1}-y_{2}\right\|
$$

then

$$
\forall t \in[0, T), d_{1}(t) \geq d_{1}(0) \mathrm{e}^{-(3 \ell t) / 2}
$$


and

$$
\forall t \in[0, T), d_{2}(t) \geq d_{2}(0) \mathrm{e}^{-(3 \ell t) / 2},
$$

where $\ell$ is the constant given by (6-iii).

Proof. The proof runs as the proof of Theorem 3.1 of [9].

\section{Approximation}

\subsection{Definition of consistency and convergence}

Let $\mathbf{T}_{h}$ be an approximation scheme that is a function from the set of the compact sets to itself with $h>0$ a scale parameter.

Definition 3.1. We say that $\mathbf{T}_{h}$ is consistent with the front propagation $H$ if the three conditions are satisfied

- There is some constant $\rho>0$, such that, for any compact $K \subset \mathbb{R}^{N}$,

$$
\sup _{y \in \mathbf{T}_{h}(K)}\|y\| \leq \rho h+(1+\rho h) \sup _{y \in K}\|y\|
$$

- for any compact set $K, \forall \phi \in \mathcal{C}^{2}\left(\mathbb{R}^{N}\right)$ and $x$ a local maximum of $\phi$ on $\mathbf{T}_{h}(K)$, we have

$$
\sup _{y \in K} \frac{\phi(y)-\phi(x)}{h} \geq H_{*}\left(x, K, \phi_{x}(x), \phi_{x x}(x)\right)+o(1),
$$

- for any compact set $K, \forall \phi \in \mathcal{C}^{2}\left(\mathbb{R}^{N}\right)$ and $x$ a local maximum of $\phi$ on $\overline{\mathbb{R}^{N} \backslash \mathbf{T}_{h}(K)}$, we have

$$
\inf _{y \in \mathbb{R}^{N} \backslash K}-\frac{\phi(y)-\phi(x)}{h} \leq H^{*}\left(x, K,-\phi_{x}(x),-\phi_{x x}(x)\right)+o(1),
$$

and $o(1)=o\left(h, x, K, \phi_{x}(x), \phi_{x x}(x)\right)$ tends to 0 locally uniformly with respect to all its arguments when $h \rightarrow 0^{+}$.

Here $H_{*}$ and $H^{*}$ differ from $H$ only if $\phi_{x}(x)=0$. In this case,

$$
H_{*}(x, K, 0, X)=\liminf _{\left(x^{\prime}, p, X^{\prime}\right) \rightarrow(x, 0, X), p \neq 0} H\left(x^{\prime}, K, p, X^{\prime}\right)
$$

and

$$
H^{*}(x, K, 0, X)=\limsup _{\left(x^{\prime}, p, X^{\prime}\right) \rightarrow(x, 0, X), p \neq 0} H\left(x^{\prime}, K, p, X^{\prime}\right)
$$

Given a compact set $K_{0}$, we iterate the operator $\mathbf{T}_{h}$ and we define

$$
K(n h)=\mathbf{T}_{h}^{n}\left(K_{0}\right)
$$

Then we note

$$
\mathcal{K}_{h}=\bigcup_{n \in \mathbb{N}} K(n h) \times\{n h\}
$$


and

$$
\mathcal{A}_{h}=\bigcup_{n \in \mathbb{N}} \overline{\mathbb{R}^{N} \backslash K(n h)} \times\{n h\} .
$$

Let us recall that the upper limit of sets $A_{h}$ when $h \rightarrow 0^{+}$is the set of cluster points of sequences of $A_{h}$ when $h \rightarrow 0$. The upper limit and the lower limit of the approximate solution $\mathcal{K}_{h}(n h)$ are defined as follows: For all $t \geq 0$

$$
\mathcal{K}^{*}(t)=\limsup _{n h \rightarrow t} \mathcal{K}_{h}(n h),
$$

and

$$
\mathcal{K}_{*}(t)=\mathbb{R}^{N} \backslash\left(\limsup _{n h \rightarrow t} \mathcal{A}_{h}(n h)\right)
$$

It can be easily checked that

$$
\forall t \geq 0, \mathcal{K}_{*}(t) \subset \mathcal{K}^{*}(t) .
$$

Theorem 3.2. Assume that the motion $H$ satisfies assumption (6) and that $\mathbf{T}_{h}$ is consistent with $H$. Given a compact set $K_{0}$ and $\epsilon>0$, let $\left(\mathcal{K}_{1}, \mathcal{K}_{2}\right)$ be a sub/super pair of solutions for $\left(K_{0}-\epsilon B, K_{0}+\epsilon B\right)$.

If $\mathcal{K}_{1}(t), \mathcal{K}_{2}(t)$ are non empty for $t \in[0, T]$, for some $T>0$, then

$$
\forall t \in[0, T], \mathcal{K}_{1}(t) \subset \operatorname{Int}\left(\mathcal{K}_{*}(t)\right) \text { and } \mathcal{K}^{*}(t) \subset \operatorname{Int}\left(\mathcal{K}_{2}(t)\right)
$$

Corollary 3.3. Assume that $\mathbf{T}_{h}$ is an approximation scheme consistent with $H$.

1. Assume that $H$ satisfies assumptions (6), (H1) and (H2). If $\mathcal{K}_{r}$ is a classical solution (with a $\mathcal{C}^{3}$ regularity) to the front propagation problem on $[0, T]$ (for some $T>0$ ) starting from $K_{0}$, then $\mathcal{K}_{h}$ converges to $\mathcal{K}_{r}$ on $[0, T]$ when $h \rightarrow 0$.

2. If $H$ is non increasing with respect to $K$ and satisfies assumption (6), then $\mathcal{K}_{*}$ contains the minimal solution $s\left(K_{0}\right)$ and $\mathcal{K}^{*}$ is contained in the maximal solution $S\left(K_{0}\right)$ :

$$
s\left(K_{0}\right) \subset \mathcal{K}_{*} \subset \mathcal{K}^{*} \subset S\left(K_{0}\right) .
$$

Remark. Let us recall that, when $H$ is non increasing with respect to $K$, we have "often" the equality between the closure of the minimal solution $\overline{s\left(K_{0}\right)}$ and the maximal one $S\left(K_{0}\right)$ (see [9]). In this case, the numerical scheme converges.

Proof of Corollary 3.3.

1. Let $\left(\mathcal{K}_{1}^{\epsilon}, \mathcal{K}_{2}^{\epsilon}\right)$ be the extremal pair of solutions with initial positions $\left(K_{0}-\epsilon B, K_{0}+\epsilon B\right)$. From Theorem 3.2, we have:

$$
\forall t \in[0, T], \mathcal{K}_{1}^{\epsilon}(t) \subset \operatorname{Int}\left(\mathcal{K}_{*}(t)\right) \text { and } \mathcal{K}^{*}(t) \subset \operatorname{Int}\left(\mathcal{K}_{2}^{\epsilon}(t)\right) .
$$

Then Theorem 2.10 asserts that $\mathcal{K}_{1}^{\epsilon}$ and $\mathcal{K}_{2}^{\epsilon}$ converge to $\mathcal{K}_{r}$ on $[0, T]$ when $\epsilon \rightarrow 0$. Thus, letting $\epsilon$ tend to 0 , we deduce the convergence of $\mathcal{K}_{h}$ to $\mathcal{K}_{r}$ on $[0, T]$ when $h \rightarrow 0$.

2. The pair $\left(S\left(K_{0}-\epsilon B\right), S\left(K_{0}+\epsilon B\right)\right)$ is a sub/super pair of solutions for $\left(K_{0}-\epsilon B, K_{0}+\epsilon B\right)$ (Prop. 2.9). From Theorem 3.2, we deduce the following inequalities:

$$
S\left(K_{0}-\epsilon B\right) \subset \mathcal{K}_{*} \subset \mathcal{K}^{*} \subset S\left(K_{0}+\epsilon B\right) .
$$


Since these inclusions hold true for any $\epsilon>0$, we let $\epsilon \rightarrow 0^{+}$, and we get, thanks to Proposition 2.9 again, that

$$
s\left(K_{0}\right) \subset \mathcal{K}_{*} \subset \mathcal{K}^{*} \subset S\left(K_{0}\right)
$$

\section{Proof of Theorem 3.2.}

1. Lemma 3.4. If $H$ satisfies the assumptions (6), then $\mathcal{K}^{*}(t)$ and $\mathcal{K}_{*}(t)$ are bounded for $t \in[0, T]$.

Proof. Let us set

$$
d_{h}(n h)=\sup _{y \in K_{h}(n h)}\|y\| .
$$

We have to estimate $\lim \sup _{h \rightarrow 0} \sup _{n h \leq T} d_{h}(n h)$. Thanks to the first property of consistency, we have

$$
\forall n \geq 0, d_{h}(n h) \leq(1+\rho h)^{n}-1+d_{h}(0)(1+\rho h)^{n} .
$$

Hence

$$
\limsup _{h \rightarrow 0} \sup _{n h \leq T} d_{h}(n h) \leq \mathrm{e}^{\rho T}-1+\mathrm{e}^{\rho T} \sup _{y \in K_{0}}\|y\| .
$$

2. Lemma 3.5. For any $x_{0} \in \mathbb{R}^{N}$ the functions $d_{\mathcal{K}^{*}(.)}\left(x_{0}\right)$ and $d_{\widehat{\mathcal{K}}_{*}(.)}\left(x_{0}\right)$ are lower semi-continuous on the left on $(0, T)$, in the sense that, for any $t \in(0, T)$, we have

$$
\liminf _{s \rightarrow t^{-}} d_{\mathcal{K}^{*}(s)}\left(x_{0}\right) \geq d_{\mathcal{K}^{*}(t)}\left(x_{0}\right)
$$

and

$$
\liminf _{s \rightarrow t^{-}} d_{\widehat{\mathcal{K}_{*}}(s)}\left(x_{0}\right) \geq d_{\widehat{\mathcal{K}_{*}}(t)}\left(x_{0}\right)
$$

Proof. We only prove the first inequality, the proof for the second one involving the same arguments. Assume that, contrary to our claim,

$$
\liminf _{s \rightarrow t^{-}} d_{\mathcal{K}^{*}(s)}\left(x_{0}\right)<d_{\mathcal{K}^{*}(t)}\left(x_{0}\right) .
$$

Then, there is some $\bar{x} \in \mathcal{K}^{*}(t)$ and some $\epsilon>0$ with

$$
\forall s \in(t-\epsilon, t), \quad B(\bar{x}, \epsilon) \cap \mathcal{K}^{*}(s)=\emptyset .
$$

Therefore, for $\epsilon>0$, the map

$$
\phi^{\epsilon}(s, y)=-s-\epsilon\|y-\bar{x}\|^{2}
$$

has a local strict maximum on $\mathcal{K}^{*}$ at $(t, \bar{x})$. From standard arguments, there exists a sequence $\left(n_{h} h, x_{h}\right)$ converging towards $(t, \bar{x})$ such that $\left(n_{h} h, x_{h}\right)$ is a local maximum on $\mathcal{K}_{h}$ for $\phi^{\epsilon}$.

Since $\mathbf{T}_{h}$ is consistent

$$
\sup _{y \in \mathcal{K}_{h}\left(\left(n_{h}-1\right) h\right)} \frac{\phi^{\epsilon}\left(n_{h} h, y\right)-\phi^{\epsilon}\left(n_{h} h, x_{h}\right)}{h} \geq H_{*}\left(x_{h}, \mathcal{K}_{h}\left(n_{h} h-h\right), \phi_{x}^{\epsilon}\left(n_{h} h, x_{h}\right), \phi_{x x}^{\epsilon}\left(n_{h} h, x_{h}\right)\right)+o(1) .
$$


Hence

$$
\sup _{y \in \mathcal{K}_{h}\left(\left(n_{h}-1\right) h\right)} \frac{\phi^{\epsilon}\left(n_{h} h, y\right)-\phi^{\epsilon}\left(n_{h} h, x_{h}\right)}{h} \geq \epsilon H_{*}\left(x_{h}, \mathcal{K}_{h}\left(n_{h} h-h\right),-2\left(x_{h}-\bar{x}\right),-2 I\right)+o(1) .
$$

On the other hand, from assumption (6), for any $M>0$ and $\gamma>0$, there exists a constant $M^{\prime}$ such that

$$
|H(y, K, p,-2 I)| \leq M^{\prime}
$$

for any $(x, K, p, A)$ such that

$$
\|y-\bar{x}\| \leq \gamma,\|p\| \leq M, \sup _{y \in K}\|y\| \leq M
$$

Then we choose $\epsilon=\frac{1}{2 M^{\prime}}$. So we have

$$
\sup _{y \in \mathcal{K}_{h}\left(\left(n_{h}-1\right) h\right)} \frac{\phi^{\epsilon}\left(n_{h} h, y\right)-\phi^{\epsilon}\left(n_{h} h, x_{h}\right)}{h} \geq-\epsilon M^{\prime}+o(1)=-\frac{1}{2}+o(1) .
$$

With the definition of $\phi^{\epsilon}$, for any $y \in \mathcal{K}_{h}\left(\left(n_{h}-1\right) h\right)$, we have

$$
\frac{\phi^{\epsilon}\left(n_{h} h, y\right)-\phi^{\epsilon}\left(n_{h} h, x_{h}\right)}{h}=\frac{\phi^{\epsilon}\left(\left(n_{h}-1\right) h, y\right)-\phi^{\epsilon}\left(n_{h} h, x_{h}\right)}{h}-1 \text {. }
$$

As $\left(n_{h} h, x_{h}\right)$ is a local maximum on $\mathcal{K}_{h}$ for $\phi^{\epsilon}$, we deduce

$$
\frac{\phi^{\epsilon}\left(n_{h} h, y\right)-\phi^{\epsilon}\left(n_{h} h, x_{h}\right)}{h} \leq-1
$$

Hence

$$
\sup _{y \in \mathcal{K}_{h}\left(\left(n_{h}-1\right) h\right)} \frac{\phi^{\epsilon}\left(n_{h} h, y\right)-\phi^{\epsilon}\left(n_{h} h, x_{h}\right)}{h} \leq-1
$$

This is in contradiction with (14).

3. Next we prove that

$$
K_{0} \subset \mathcal{K}^{*}(0) \text { and } \mathcal{K}_{*}(0) \subset K_{0}
$$

Proof. We only prove the first inclusion, the proof for the other being similar.

We argue by contradiction. Assume that there is some $x \in \mathcal{K}^{*}(0) \backslash K_{0}$. Then, for any $\epsilon>0$, the map

$$
\phi(s, y)=-\epsilon\|y-x\|^{2}-s
$$

has a strict local maximum on $\mathcal{K}^{*}$ at $(0, x)$. From the definition of $\mathcal{K}^{*}$, there are $n_{h} h \rightarrow 0^{+}, x_{h} \in \mathcal{K}_{h}(n h)$ converging to $x$, local maxima of $\phi$ on $\mathcal{K}_{h}$. Note that $n_{h} h>0$ because $\mathcal{K}_{h}(0)=K_{0}$ and $x_{h} \notin K_{0}$ for $h$ sufficiently small, since $x_{h} \rightarrow x \notin K_{0}$.

From the consistency assumption, we have

$$
\sup _{y \in \mathcal{K}_{h}\left(\left(n_{h}-1\right) h\right)} \phi\left(n_{h} h, y\right) \geq \phi\left(n_{h} h, x_{h}\right)+h H^{*}\left(x_{h}, \mathcal{K}_{h}\left(n_{h} h\right),-\epsilon\left(x_{h}-x\right),-\epsilon I_{N}\right)+h o(1) .
$$


We can always choose $\epsilon>0$ at the beginning of the proof in such a way that

$$
\left|H^{*}\left(x_{h}, \mathcal{K}_{h}(n h),-\epsilon\left(x_{h}-x\right),-\epsilon I_{N}\right)\right| \leq 1 / 4
$$

thanks to assumption (6,iii). Moreover, since the $\mathcal{K}_{h}\left(n_{h} h\right)$ are bounded, we can also choose $h$ sufficiently small in such a way that the $|o(1)|$ is smaller than $1 / 4$. Thus we have

$$
\sup _{y \in \mathcal{K}_{h}\left(\left(n_{h}-1\right) h\right)} \phi\left(n_{h} h, y\right) \geq \phi\left(n_{h} h, x_{h}\right)-h / 2
$$

Since $\phi$ has a local maximum on $\mathcal{K}_{h}$ at $\left(n_{h} h, x_{h}\right)$, we have

$$
\forall y \in \mathcal{K}_{h}\left(\left(n_{h}-1\right) h\right), \phi\left(n_{h} h, y\right)=\phi\left(\left(n_{h}-1\right) h, y\right)-h \leq \phi\left(n_{h} h, x_{h}\right)-h
$$

Hence there is a contradiction.

4. Let us set

$$
T^{*}:=\sup \left\{t \in[0, T] \mid \begin{array}{l}
\forall s \in[0, t), \mathcal{K}_{1}(t) \subset \operatorname{Int}\left(\mathcal{K}_{*}(t)\right) \\
\text { and } \mathcal{K}^{*}(t) \subset \operatorname{Int}\left(\mathcal{K}_{2}(t)\right)
\end{array}\right\} .
$$

We have to prove that $T^{*}=T$.

5. We claim that, since $\mathbf{T}_{h}$ is consistent with $H$, then conditions (C1), (C2) and (C3) of Theorem 2.11 are fulfilled.

We postpone for a while the proof of this claim and complete the proof of the Theorem. Thanks to this result, we have from Theorem 2.11

$$
\forall t \in\left[0, T^{*}\right), d_{1}(t) \geq d_{1}(0) \mathrm{e}^{-(3 \ell t) / 2}
$$

and

$$
\forall t \in\left[0, T^{*}\right), d_{2}(t) \geq d_{2}(0) \mathrm{e}^{-(3 \ell t) / 2}
$$

where $d_{1}$ and $d_{2}$ are defined by (12) and (13).

Since Lemma 3.5 asserts that $d_{1}$ and $d_{2}$ are continuous on the left on $[0, T]$, we have for $i=1,2$

$$
\forall t \in\left[0, T^{*}\right], d_{i}(t) \geq d_{i}(0) \mathrm{e}^{-(3 \ell t) / 2} .
$$

Thus we have for $i=1,2$ and $\alpha>0$ small enough

$$
d_{i}\left(T^{*}\right) \geq d_{i}(0) \mathrm{e}^{-\left(3 \ell T^{*}\right) / 2}>(1-\alpha) d_{i}(0) \mathrm{e}^{-3 \ell T^{*}}
$$

because $d_{i}(0)>0$. From the lower semi-continuity of $d_{i}$ there is some $\tau>0$ with

$$
\forall t \in\left[T^{*}, T^{*}+\tau\right), d_{i}(t) \geq(1-\alpha) d_{i}(0) \mathrm{e}^{-3 \ell t}>0 .
$$

This is in contradiction with the definition of $T^{*}$. Hence we have $T^{*}=T$.

6. Lemma 3.6. If $\mathcal{K}^{*}$ satisfies the external condition for $H^{b}$ for any test function of the form $\phi(t, x)=$ $f(x)+g(t)$, then $\mathcal{K}^{*}$ satisfies the external condition for $H^{b}$.

The proof is quite standard [19], so we only give it in Appendix. 
7. We now prove that $\mathcal{K}^{*}$ satisfies the external condition for $H^{b}$ on $\left[0, T^{*}\right]$. Since the proof that $\mathcal{K}_{*}$ satisfies the internal condition for $H^{\sharp}$ on $\left[0, T^{*}\right]$ is similar, we omit it.

Let $(\bar{t}, \bar{x}) \in \partial \mathcal{K}^{*}$ be a maximum on $\mathcal{K}^{*}$ for a test function $\phi(t, x)=f(x)+g(t)$. Then, there exists a sequence $\left(n_{h} h, x_{h}\right)$ converging towards $(\bar{t}, \bar{x})$ such that $\left(n_{h} h, x_{h}\right)$ is a local maximum on $\mathcal{K}_{h}$ for $\phi$.

The function $f$ has a local maximum on $K_{h}\left(n_{h} h\right)$ at $x_{h}$. Since $\mathbf{T}_{h}$ is consistent

$$
\sup _{y \in \mathcal{K}_{h}\left(\left(n_{h}-1\right) h\right)} \frac{f(y)-f\left(x_{h}\right)}{h} \geq H_{*}\left(x_{h}, \mathcal{K}_{h}\left(n_{h} h-h\right), \phi_{x}\left(n_{h} h, x_{h}\right), \phi_{x x}\left(n_{h} h, x_{h}\right)\right)+o(1) .
$$

From the definition of $H^{b}$ and since

$$
\mathcal{K}_{*}\left(n_{h} h-h\right)-o(h) B \subset \mathcal{K}_{h}\left(n_{h} h-h\right),
$$

and

$$
\mathcal{K}_{h}\left(n_{h} h-h\right) \subset \mathcal{K}^{*}\left(n_{h} h-h\right)+o(h) B,
$$

we get, if we set $t_{h}=n_{h} h, \phi_{x}=\phi_{x}\left(t_{h}, x_{h}\right), \phi_{x x}=\phi_{x x}\left(t_{h}, x_{h}\right)$,

$$
H_{*}\left(x_{h}, \mathcal{K}_{h}\left(t_{h}-h\right), \phi_{x}, \phi_{x x}\right) \geq H_{*}^{\mathrm{b}}\left(x_{h}, \mathcal{K}_{*}\left(t_{h}-h\right)-o(h) B, \mathcal{K}^{*}\left(t_{h}-h\right)+o(h) B, \phi_{x}, \phi_{x x}\right) .
$$

On the other hand, $\left(t_{h}, x_{h}\right)$ is a local maximum on $\mathcal{K}_{h}$ for $\phi$. Thus

$$
\forall y \in \mathcal{K}_{h}\left(t_{h}-h\right), f(y)+g\left(t_{h}-h\right) \leq f\left(x_{h}\right)+g\left(t_{h}\right) .
$$

Therefore, $\forall y \in \mathcal{K}_{h}\left(t_{h}-h\right)$,

$$
f(y)-f\left(x_{h}\right) \leq g\left(t_{h}\right)-g\left(t_{h}-h\right)=h g_{t}\left(t_{h}-h\right)+o(h),
$$

so we deduce, from (15) and (16), that

$$
\phi_{t}\left(t_{h}, x_{h}\right)+o(1) \geq H_{*}^{\mathrm{b}}\left(x_{h}, \mathcal{K}_{*}\left(t_{h}-h\right)-o(h) B, \mathcal{K}^{*}\left(t_{h}-h\right)+o(h) B, \phi_{x}, \phi_{x x}\right) .
$$

When $h$ tends to 0 , we get the external condition.

\subsection{Examples}

\subsubsection{Thinning of a shape}

We present a motion which formalizes a thinning of the initial shape and intends to compute a kind of skeleton of the shape. Blum [7] actually defined the skeleton in the following way: If fire is turned on at the boundary of the shape, and it travels with uniform speed, the skeleton is the points were two or more fire fronts collapse. We give an example of dynamics $h$ which does not compute the true skeleton defined by Blum but rather corresponds to a thinning evolution in dimension 2 with a smoothing effect.

The evolution equation is

$$
V=\left(\left[\mathbf{k}_{x}\right]_{-}-1\right) \rho^{2}\left(x, K_{t}\right)
$$

i.e., $h$ is defined by

$$
h(x, K, p, A)=\left([\operatorname{Trace}(A)]_{-}-1\right) \rho^{2}(x, K)
$$


where, for any $r \in \mathbb{R}$, we have set $[r]_{-}=\min (0, r)$. The term $[\operatorname{Trace} A]_{-}$is the negative part of the mean curvature. It is a regularizing term. The term -1 is an erosion. Finally, the term $\rho^{2}(x, K)$ is a stopping term which freezes the evolution when the shape becomes too thin. It is defined by

$$
\rho(x, K)=\beta\left(\sup _{\|e\|=1} \frac{\mid(B(x+\theta e, \theta) \cap K \mid}{|B(x+\theta e, \theta)|}\right),
$$

$|E|$ is the Lebesgue measure of a set $E, \theta>0$ is fixed and the function $\beta \in \mathcal{C}^{2}(\mathbb{R}, \mathbb{R})$ is Lipschitz and non decreasing and satisfies

- $\beta(s)=1$ if $s \geq 1$,

- $\beta(s)=0$ if $s<1-\eta$ for small $\eta>0$ small enough.

Let us point out that the map $h$ is decreasing with respect to $K$. Hence this motion does not satisfy the inclusion principle. However, the map $H$ defined from the dynamics $h$ by (3):

$$
H(x, K, p, X)=\left(\left[\operatorname{Trace}\left(X_{\left.\right|_{p} \perp}\right)\right]_{+}+\|p\|\right) \rho^{2}(x, K)
$$

where $[r]_{+}=\max \{0, r\}$, satisfies assumptions $(6)$.

To approximate the evolution, we consider the operator $\mathbf{T}_{h}$

$$
\mathbf{T}_{h}(K)=\left\{\begin{array}{ll}
x \in K \mid & \begin{array}{l}
\exists B \in \mathcal{B}_{h}, \forall b \in B, \forall\left\|b^{\prime}\right\| \leq h, \\
x+\rho(x, K) b+\rho^{2}(x, K) b^{\prime} \in K
\end{array}
\end{array}\right\},
$$

where the family $\mathcal{B}_{h}$, already used in [11], is the family of the segments of length $2 \sqrt{2 h}$ with center the origin $O$. This family $\mathcal{B}_{h}$ satisfies the following property: For any function $u \in \mathcal{C}^{2}\left(\mathbb{R}^{2}\right)$ with $D u \neq 0$

$$
\inf _{B \in \mathcal{B}_{h}} \sup _{y \in B} u(x+y)=u(x)+h\left[\operatorname{Trace} D^{2} u_{\left.\right|_{D u \perp}}\right]_{+}+o(h) .
$$

(For the proof, see [11].)

Lemma 3.7. The operator $\mathbf{T}_{h}$ is consistent with $H$.

Proof. Let $K$ be a compact subset of $\mathbb{R}^{N}$. Since we clearly have $\mathbf{T}_{h}(K) \subset K$, the first property of consistency is satisfied.

We only check the second property, the proof for the third one being similar. Assume that $\phi \in \mathcal{C}^{2}\left(\mathbb{R}^{N}\right)$ has a local maximum at $x_{h} \in \mathbf{T}_{h}(K)$. We first assume that $\phi_{x}\left(x_{h}\right) \neq 0$. Then we set

$$
M=\sup _{y \in K} \frac{\phi(y)-\phi\left(x_{h}\right)}{h}
$$

We have to prove

$$
M \geq H_{*}\left(x_{h}, K, \phi_{x}\left(x_{h}\right), \phi_{x x}\left(x_{h}\right)\right)+o(1) .
$$

Since $x_{h} \in \mathbf{T}_{h}(K)$, there exists some $B \in \mathcal{B}_{h}$ such that, for any $b \in B$ and for any $\left|b^{\prime}\right| \leq h$, the point $\left.y=x_{h}+\rho\left(x_{h}, K\right)\right) b+\rho^{2}\left(x_{h}, K\right) b^{\prime}$ belongs to $K$. Therefore

$$
M \geq \frac{\phi\left(x_{h}+\rho\left(x_{h}, K\right) b+\rho^{2}\left(x_{h}, K\right) b^{\prime}\right)-\phi\left(x_{h}\right)}{h} .
$$


With a Taylor expansion, we deduce

$$
M h \geq \rho\left(x_{h}, K\right) \phi_{x}\left(x_{h}\right) \cdot b+\rho^{2}\left(x_{h}, K\right) \phi_{x}\left(x_{h}\right) b^{\prime}+\frac{1}{2} \rho^{2}\left(x_{h}, K\right) \phi_{x x}\left(x_{h}\right) b b+o(h) .
$$

Hence

$$
M h \geq \rho\left(x_{h}, K\right) \inf _{B \in \mathcal{B}_{h}} \sup _{b \in B}\left(\phi_{x}\left(x_{h}\right) b+\frac{1}{2} \rho\left(x_{h}, K\right) \phi_{x x}\left(x_{h}\right) b b\right)+\rho^{2}\left(x_{h}, K\right) \sup _{\left|b^{\prime}\right| \leq h}\left(\phi_{x}\left(x_{h}\right) b^{\prime}\right)+o(h) .
$$

Because of (18) and the fact that $\phi_{x}\left(x_{h}\right) \neq 0$, we get

$$
M \geq \rho^{2}\left(x_{h}, K\right)\left(\left[\operatorname{Trace} D^{2} \phi_{\left.\right|_{D_{\phi}}{ }^{+}}\right]^{+}+\|D \phi\|\right)+o(1) .
$$

Let us now assume that $\phi_{x}\left(x_{h}\right)=0$. Then, with the same computation as above, we have

$$
M h \geq \frac{1}{2} \rho^{2}\left(x_{h}, K\right) \inf _{B \in \mathcal{B}_{h}} \sup _{b \in B} \phi_{x x}\left(x_{h}\right) b b+o(h) .
$$

If we denote by $\lambda$ the smallest eigenvalue of $\phi_{x x}\left(x_{h}\right)$, we have therefore

$$
M \geq \rho^{2}\left(x_{h}, K\right)[\lambda]_{+}+o(1) \geq H_{*}\left(x_{h}, K, 0, \phi_{x x}\left(x_{h}\right)\right)+o(1) .
$$

Hence we get the consistency.

We now describe the implementation of the algorithm. The scheme $\mathbf{T}_{h}$ is rather difficult to implement as such, merely because it is not easy to take into account the difference between $h$ and $\sqrt{h}$ (in practice, one would like to take $h=1$ !). In order to overcome this difficulty, we alternate $n$ inf-sup scheme $T_{1}$ with one erosion $T_{2}$ and we repeat this algorithm until we get the skeleton. The parameter $n$ is a scale parameter. Roughly speaking, we have $n h \approx \sqrt{h}$. For the experiments, $n=2$, i.e., $h \approx 1 / 4$.

Therefore the scheme $T$ is of the form

$$
T=T_{2} \circ\left(T_{1}\right)^{n}
$$

where

$$
\mathbf{T}_{1}(K)=\left\{x \in K \mid \exists B \in \mathcal{B}_{1}, \forall b \in B, x+\rho(x, K) b \in K\right\},
$$

and

$$
\mathbf{T}_{2}(K)=\left\{x \in K \mid \forall\left\|b^{\prime}\right\|_{\infty} \leq 1, x+\rho^{2}(x, K) b^{\prime} \in K\right\},
$$

and where, for any $x \in K$, the function $\rho(x, K)$ is computed in the following way:

$$
\rho(x, K)=\beta\left(\sup _{\|e\|_{\infty}=1} \frac{\operatorname{Vol}((B(x+e, 1) \cap K)}{\operatorname{Vol}(B(x+e, 1))}\right),
$$

$\operatorname{Vol}(E)$ is the number of pixels in $E, B(y, r)=\left\{z \in\right.$ grid $\left.\mid\|y-z\|_{\infty} \leq r\right\}$, and the function $\beta$ is defined by

- $\beta(s)=1$ if $s \geq 1$,

- $\beta(s)=0$ if $s<1$.

We make a comparison with a thinning algorithm created by Luc Vincent [27]. This algorithm needs to smooth the shapes before the skeletonization, and a pruning after. The results are on the Figure 2. 

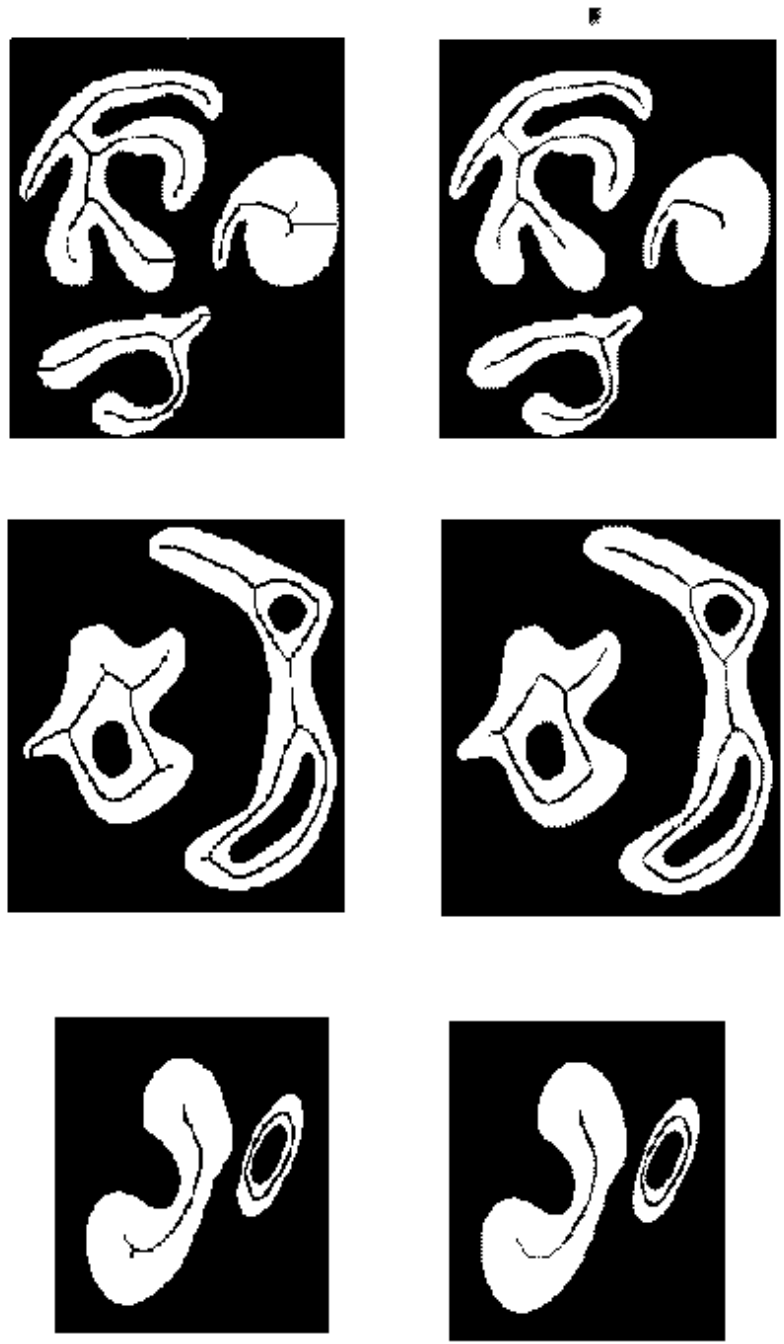

FiguRE 2. We have the skeletons with a thinning algorithm (Luc Vincent) in the left column and with the algorithm we present on the right column.

\subsubsection{A scheme for equation (1)}

We now investigate a numerical scheme for the motion given by

$$
V=\mathbf{k}_{x}+\alpha+\beta\left|K_{t}\right|,
$$

i.e., when

$$
h(K, p, A)=\operatorname{Trace}(A)+\alpha+\beta|K| .
$$

Then the associated map $H$ is given by

$$
H(K, p, X)=\operatorname{Trace}\left(X_{\left.\right|_{p} \perp}\right)-(\alpha+\beta|K|)\|p\| .
$$


As already pointed out, $H$ satisfies the conditions (6). Moreover, the associated evolution satisfies the inclusion principle if and only if $\beta \geq 0$.

To approximate the evolution, we introduce the operator $\mathbf{T}_{h}$ defined by

- If $(\alpha+\beta|K|) \geq 0$, then

$$
\mathbf{T}_{h}(K)=\left\{\begin{array}{ll}
x \in K \mid & \begin{array}{l}
\exists B \in \mathcal{B}_{h}, \forall b \in B, \exists\left\|b^{\prime}\right\| \leq h(\alpha+\beta|K|), \\
x+b+b^{\prime} \in K
\end{array}
\end{array}\right\} .
$$

- If $(\alpha+\beta|K|) \leq 0$, then

$$
\mathbf{T}_{h}(K)=\left\{\begin{array}{ll}
x \in K \mid & \begin{array}{l}
\exists B \in \mathcal{B}_{h}, \forall b \in B, \forall\left\|b^{\prime}\right\| \leq h|\alpha+\beta| K||, \\
x+b+b^{\prime} \in K
\end{array}
\end{array}\right\}
$$

where, now, the family $\mathcal{B}_{h}$ is the family of the extremities of the segments of length $2 \sqrt{2 h}$ with center the origin O. Namely:

$$
\mathcal{B}_{h}=\left\{\{\sqrt{2 h} w,-\sqrt{2 h} w\} \subset \mathbb{R}^{2} \times \mathbb{R}^{2} \mid w \in \mathbb{R}^{2},\|w\|=1\right\} .
$$

It can be proved as in [11] that this family $\mathcal{B}_{h}$ satisfies the following property: For any function $u \in \mathcal{C}^{2}\left(\mathbb{R}^{2}\right)$ with $D u \neq 0$

$$
\inf _{B \in \mathcal{B}_{h}} \sup _{y \in B} u(x+y)=u(x)+h \operatorname{Trace}\left(D^{2} u_{\left.\right|_{D u} \perp}\right)+o(h) .
$$

Lemma 3.8. The operator $\mathbf{T}_{h}$ is consistent with $H$.

Proof. It is the same as for Lemma 3.7 .

Acknowledgements. We would like to thank the anonymous referees for their comments and suggestions on the paper.

\section{APPENDIX}

In this appendix, we prove Lemma 3.6.

Proof of Lemma 3.6.

We consider a test function $\phi$ with a maximum on $\mathcal{K}^{*}$ at some point $(\bar{t}, \bar{x}) \in \partial \mathcal{K}^{*}$. Without loss of generality, we may assume that the origin is at $(\bar{t}, \bar{x})$, so that $(\bar{t}, \bar{x})=(0,0)$. We have the Taylor expansion

$$
\phi(t, x)=\phi(0,0)+\phi_{x}(0,0) x+\phi_{t}(0,0) t+\frac{1}{2} \phi_{x x}(0,0) x x+\frac{1}{2} \phi_{t t}(0,0) t^{2}++t \phi_{t x}(0,0) x+o\left(\|x\|^{2}+|t|^{2}\right) .
$$

We set

$$
f(x)=\phi(0,0)+\phi_{x}(0,0) x+\frac{1}{2} \phi_{x x}(0,0) x x-\epsilon\|x\|^{2}-\epsilon\left|\phi_{t x}(0,0)\right|\|x\|^{2},
$$

and

$$
g(t)=\phi_{t}(0,0) t-\frac{\left|\phi_{t x}(0,0)\right|}{\epsilon} t^{2}-\epsilon t^{2}+\frac{1}{2} \phi_{t t}(0,0) t^{2}
$$


So that

$$
\phi(t, x)=f(x)+g(t)+\epsilon\left|\phi_{t x}(0,0)\right|\|x\|^{2}+\frac{\left|\phi_{t x}(0,0)\right|}{\epsilon} t^{2}+t \phi_{t x}(0,0) x+\epsilon\left(\|x\|^{2}+t^{2}\right)+o\left(\|x\|^{2}+|t|^{2}\right) .
$$

Since

$$
\epsilon\left|\phi_{t x}(0,0)\right| \|\left. x\right|^{2}+\frac{\left|\phi_{t x}(0,0)\right|}{\epsilon} t^{2}+t \phi_{t x}(0,0) x+\epsilon\left(\|x\|^{2}+t^{2}\right)+o\left(\|x\|^{2}+|t|^{2}\right) \geq 0,
$$

for $(t, x)$ small enough, we have for $(t, x)$ close to $(0,0)$

$$
f(x)+g(t) \leq \phi(t, x) .
$$

Then $f(x)+g(t)$ has a maximum on $\mathcal{K}^{*}$ at $(0,0) \in \partial \mathcal{K}^{*}$. By assumption

$$
H_{*}^{\mathrm{b}}\left(0, \mathcal{K}_{*}(0), \mathcal{K}^{*}(0), f_{x}(0), f_{x x}(0)\right) \leq g_{t}(0) .
$$

As $f_{x}(0)=\phi_{x}(0,0), f_{x x}(0)=\phi_{x x}(0,0)-2 \epsilon\left(1+\left|\phi_{t x}(0,0)\right|\right) I_{N}$ and $g_{t}(0)=\phi_{t}(0,0)$, we deduce

$$
H_{*}^{b}\left(0, \mathcal{K}_{*}(0), \mathcal{K}^{*}(0), \phi_{x}(0,0), \phi_{x x}(0,0)-2 \epsilon\left(1+\left|\phi_{t x}(0,0)\right|\right) I_{N}\right) \leq \phi_{t}(0,0) .
$$

We let $\epsilon$ tend to 0 , then we get the result.

\section{REFERENCES}

[1] L. Alvarez, F. Guichard, P.L. Lions and J-.M. Morel, Axioms and fundamental equations of image processing. Arch. Ration. Mech. Anal. 123 (1993) 199-257.

[2] L. Ambrosio, Geometric evolution problems, distance function and viscosity solutions, in Calculus of variations and partial differential equations. Topics on geometrical evolution problems and degree theory, G. Buttazzo et al. Eds., Based on a summer school, Pisa, Italy, September 1996. Springer, Berlin (2000) 5-93; 327-337 .

[3] G. Barles and C. Georgelin, A simple proof of convergence for an approximation scheme for computing motions by mean curvature. SIAM J. Numer. Anal. 32 (1995) 484-500.

[4] G. Barles and P.M. Souganidis, Convergence of approximation schemes for fully nonlinear second order equations. Asymptotic Analysis 4 (1991) 271-283.

[5] G. Barles, H.M. Soner and P.M. Souganidis, Front propagation and phase field theory. SIAM J. Control Optim. 31 (1993) 439-469.

[6] G. Barles and P.M. Souganidis, A new approach to front propagation problems: theory and applications. Arch. Ration. Mech. Anal. 141 (1998) 237-296.

[7] H. Blum, Biological shape and visual science. J. Theor. Biology 38 (1973) 205-287.

[8] J. Bence, B. Merriman and S. Osher, Diffusion motion generated by mean curvature. CAM Report 92-18. Dept of Mathematics. University of California Los Angeles (1992).

[9] P. Cardaliaguet, On front propagation problems with nonlocal terms. Adv. Differential Equation 5 (1999) 213-268.

[10] F. Cao, Partial differential equations and mathematical morphology. J. Math. Pures Appl. 77 (1998) 909-941.

[11] F. Catte, F. Dibos and G. Koepfler, A morphological scheme for mean curvature motion. SIAM J. Numer. Anal. 32 (1995) $1895-1909$

[12] Y. Chen, Y. Giga and S. Goto, Uniqueness and existence of viscosity solutions of generalized mean curvature flow equations. J. Differential Geom. 33 (1991) 749-786.

[13] X. Chen, D. Hilhorst and E. Logak, Asymptotic behavior of solutions of an Allen-Cahn equation with a nonlocal term. Nonlinear Anal. T.M.A. 28 (1997) 1283-1298.

[14] M. Crandall, H. Ishii and P.-L. Lions, User's guide to viscosity solution of second order partial differential equations. Bull. Amer. Math. Soc. 27 (1992) 1-67.

[15] M. Crandall and P.-L. Lions, Convergent difference schemes for nonlinear parabolic equations and mean curvature motion. Numer. Math. 75 (1996) 17-41.

[16] J. Escher and G. Simonett, Moving surfaces and abstract parabolic evolution equations. Topics in nonlinear analysis, Progr. Nonlinear Differential Equations Appl. 35, Birkhäuser, Basel (1999) 183-212.

[17] L.C. Evans and J. Spruck, Motion of level sets by mean curvature I. J. Differential Geom. 33 (1991) 635-681. 
[18] Y. Giga, S. Goto, H. Ishii and M.-H. Sato, Comparison principle and convexity preserving properties for singular degenerate parabolic equations on unbounded domains. Indiana Univ. Math. J. 40 (1990) 443-470.

[19] F. Guichard and J.M. Morel, Partial differential equation and image iterative filtering. Tutorial of ICIP 95, Washington D.C., (1995).

[20] H. Ishii, A generalization of the Bence-Merriman and Osher algorithm for motion by mean curvature, in Proceedings of the international conference on curvature flows and related topics, Levico, Italy, June 27-July 2nd 1994, A. Damlamian et al. Eds. GAKUTO Int. Ser., Math. Sci. Appl. 5, Gakkotosho, Tokyo (1995) 111-127.

[21] H. Ishii, Gauss curvature flow and its approximation, in Proceedings of the international conference on free boundary problems: theory and applications, Chiba, Japan, November 7-13 1999, N. Kenmochi Ed. GAKUTO Int. Ser., Math. Sci. Appl. 14, Gakkotosho, Tokyo (2000) 198-206.

[22] S. Osher and J.A. Sethian, Front propagation with curvature dependent speed: algorithms based on Hamilton-Jacobi formulations. J. Comp. Phys. 79 (1998) 12-49.

[23] D. Pasquignon, Computation of skeleton by PDE. IEEE-ICIP, Washington (1995).

[24] D. Pasquignon, Approximation of viscosity solution by morphological filters. ESAIM: COCV 4 (1999) 335-359.

[25] J.A. Sethian, Level set methods and fast marching methods. Evolving interfaces in computational geometry, fluid mechanics, computer vision, and materials science. Cambridge Monographs Appl. Comput. Math. 3, Cambridge University Press, Cambridge (1996).

[26] H.M. Soner, Front propagation, in Boundaries, interfaces and transitions, (Banff, AB, 1995) CRM Proc. Lect. Notes 13, Amer. Math. Soc., Providence RI (1998) 185-206.

[27] L. Vincent, Files d'attentes et algorithmes morphologiques. Thèse mines de Paris (1992).

To access this journal online: www.edpsciences.org 